\title{
LOCAL EQUILIBRIUM IN INHOMOGENEOUS STOCHASTIC MODELS OF HEAT TRANSPORT
}

\author{
PÉTER NÁNDORI
}

\begin{abstract}
We extend the duality of Kipnis Marchioro and Presutti KMP82 to inhomogeneous lattice gas systems where either the components have different degrees of freedom or the rate of interaction depends on the spatial location. Then the dual process is applied to prove local equilibrium in the hydrodynamic limit for some inhomogeneous high dimensional systems and in the nonequilibrium steady state for one dimensional systems with arbitrary inhomogeneity.
\end{abstract}

\section{INTRODUCTION}

Describing the emergence of local equilibrium in systems forced out of equilibrium is one of the main challenges of nonequilibrium statistical mechanics. Most mathematical works concern stochastic interacting particle systems as they are generally more tractable than the deterministic ones. Despite the big amount of recent work in this field, little is known for systems with spatial inhomogeneity.

The present paper extends the classical duality result of Kipnis Marchioro and Presutti [KMP82] to inhomogeneous systems, where the inhomogeneity means that either (A) the components of the system have different degrees of freedom or (B) the interaction rate between the components depends on their location. The dual process is roughly speaking a collection of biased random walkers that interact with one another once their distance is not bigger than 1 , and are completely independent otherwise. We leverage the dual process to prove the existence of local equilibrium in several cases. Some of these results were announced in [LNY15]. The systems we consider are somewhat similar to the ones recently introduced in [CGRS15], but are not the same.

1.1. Informal description. To fix notation, we denote by $\Gamma(\alpha, c)$ the probability distribution with density $\frac{x^{\alpha-1} \exp (-x / c)}{c^{\alpha} \Gamma(\alpha)}$ for $x>0$, where $\alpha>0$ is the shape parameter and $c>0$ is the scale parameter ( $\Gamma$ is the usual Gamma function). The $k$ th moment of the Gamma distribution is

$$
\int_{0}^{\infty} \frac{x^{k+\alpha-1} \exp (-x / c)}{c^{\alpha} \Gamma(\alpha)} d x=\frac{c^{k} \Gamma(\alpha+k)}{\Gamma(\alpha)}
$$


The $\operatorname{Beta}(\alpha, \beta)$ distribution with parameters $\alpha, \beta>0$ has density $\frac{\Gamma(\alpha+\beta)}{\Gamma(\alpha) \Gamma(\beta)} x^{\alpha-1}(1-$ $x)^{\beta-1}$ for $x>0$. For brevity, we will write $B(\alpha, \beta)=\frac{\Gamma(\alpha) \Gamma(\beta)}{\Gamma(\alpha+\beta)}$.

Our model can be informally described as follows. First consider two systems with degrees of freedom $\omega_{i}$ and energy $\xi_{i}$ for $i=1,2$. Now let the two systems exchange energies by a microcanonical procedure: redistribute the total energy according to the law of equipartition. Representing the energy per the $j$ th degree of freedom by $X_{j}^{2}$ for $j=1, \ldots, \omega_{1}+\omega_{2}, \vec{X}$ is thus uniformly distributed on the sphere $\left(\xi_{1}+\xi_{2}\right) \mathbb{S}^{\omega_{1}+\omega_{2}-1}$. Writing $\vec{X}=\left(\xi_{1}+\xi_{2}\right) \vec{Y} /\|\vec{Y}\|$, where $\vec{Y}$ has $\omega_{1}+\omega_{2}$ dimensional standard normal distribution, the first system's energy is updated to

$$
\xi_{1}^{\prime}=X_{1}^{2}+\ldots+X_{\omega_{1}}^{2}=\frac{\left(\xi_{1}+\xi_{2}\right)\left(Y_{1}^{2}+\ldots+Y_{\omega_{1}}^{2}\right)}{Y_{1}^{2}+\ldots+Y_{\omega_{1}+\omega_{2}}^{2}}
$$

which is well known to have $\left(\xi_{1}+\xi_{2}\right) \operatorname{Beta}\left(\omega_{1} / 2, \omega_{2} / 2\right)$ distribution.

In the simplest case, our model consists of a one dimensional chain of systems located at sites $1,2, \ldots, L-1$ and possibly having different degrees of freedom. Then we choose pairs of nearest neighbors randomly and update their energies by the above rule. Furthermore, the systems at site 1 and $L-1$ are coupled to heat baths of different temperature. Then we study the macroscopic energy propagation and the emergence of local equilibrium. The special case of constant 2 degrees of freedom is [KMP82, while systems with (arbitrary) constant degrees of freedom have been studied in [CGRS15]. A chain of alternating billiard particles (2 degrees of freedom) and pistons (1 degree of freedom), has been proposed in [BGNSzT15]. There, the energies are updated via a much more complicated deterministic rule.

Finally, we consider two more generalizations: (A) to higher dimensions and (B) to inhomogeneity in the rate of interaction.

1.2. Random walks end electrical networks. Here, we review the connection between one dimensional random walks and electrical networks as we will need them in the study of the dual process. The connection extends to much more general graphs than $\mathbb{Z}^{1}$, see e.g. [K14], Section 19. Assume that the weights (conductances) $w_{i+1 / 2}, i=A, A+1, \ldots, B$, are given and a random walker $\mathcal{S}_{k}$ is defined on the set $\{A, A+1, \ldots, B\}$ by $\mathcal{S}(0)=I$ and

$\mathbb{P}\left(\mathcal{S}_{k}=i+1 \mid S_{k}=i\right)=\frac{w_{i+1 / 2}}{w_{i-1 / 2}+w_{i+1 / 2}}, \quad \mathbb{P}\left(\mathcal{S}_{k}=i-1 \mid S_{k}=i\right)=\frac{w_{i-1 / 2}}{w_{i-1 / 2}+w_{i+1 / 2}}$

for all $i=A+1, \ldots, B-1$ (the states $A$ and $B$ are absorbing). Then the resistances $R_{i+1 / 2}=1 / w_{i+1 / 2}$ and the hitting probability

$$
p=\mathbb{P}\left(\min \left\{k: \mathcal{S}_{k}=0\right\}<\min \left\{k: \mathcal{S}_{k}=N\right\}\right)
$$


are connected by the well known formula

$$
p=\frac{\sum_{i=I}^{B-1} R_{i}}{\sum_{i=A}^{B-1} R_{i}} .
$$

Finally, to fix terminology, we denote by SSRW the simple symmetric random walk, i.e. a random walk with independent steps, uniformly distributed on the neighbors of the origin in $\mathbb{Z}^{d}$.

1.3. Organization. The rest of the paper is organized as follows. We define our model precisely in Section 2. In Section [3, the dual process is defined and the duality relation is proved. In Section 4 the main results, namely local equilibrium in the hydrodynamic limit in dimension $\geq 2$ and in the nonequilibrium steady state in dimension 1, are formulated. Section 5 contains the proofs of the theorems concerning the hydrodynamic limit, namely Theorems 1 and 2, except for the proof of Proposition 6. Then Proposition 6] is proved in Section 6 (except for the proof of Lemma 66). Section 7 is the proof of Theorem 3, which is the case of nonequilibrium steady state. Finally, the Appendix contains the proof of Lemma 6.

\section{THE MODEL}

Let us fix a dimension $d \geq 1$, and $\mathcal{D} \subset \mathbb{R}^{d}$, a bounded, connected open set for which $\partial \mathcal{D}$ is a piecewise $\mathcal{C}^{2}$ submanifold with no cusps. We prescribe a continuous function $T: \mathbb{R}^{d} \backslash \mathcal{D} \rightarrow \mathbb{R}_{+}$to be thought of as temperature. For $L \gg 1$, the physical domain of our system is

$$
\mathcal{D}_{L}=L \mathcal{D} \cap \mathbb{Z}^{d}
$$

At each lattice point $z \in \mathcal{D}_{L}$ (which will be called a site) there is a physical system of $\omega_{z}$ degrees of freedom. The rate of interaction along the edge $e=$ $(u, v) \in \mathcal{E}\left(\mathcal{D}_{L}\right)$ is denoted by $r_{e}=r_{(u, v)}$ (here, $\mathcal{E}\left(\mathcal{D}_{L}\right)$ is the set of edges of the lattice $\mathbb{Z}^{d}$ restricted to $\left.\mathcal{D}_{L}\right)$. Finally, we also fix rates of interaction between boundary sites and the heat bath: $r_{(u, v)}$ for $u \in \mathcal{D}_{L}, v \in \mathbb{Z}^{d} \backslash \mathcal{D}_{L}$ with $\|u-v\|=1$.

Throughout this paper, $|\cdot|$ denotes the cardinality of a finite set, and for $v \in \mathbb{R}^{d},\langle v\rangle$ is its closest point in $\mathbb{Z}^{d}$.

The time evolution of the energies $\boldsymbol{X}(t)=\boldsymbol{X}^{(L)}(t)=\left(\xi_{v}^{(L)}(t)\right)_{v \in \mathcal{D}_{L}}$ is a Markov process with generator

$$
(G f)(\underline{\xi})=\left(G_{1} f\right)(\underline{\xi})+\left(G_{2} f\right)(\underline{\xi}),
$$

where $G_{1}$ describes interactions within $\mathcal{D}_{L}$ and $G_{2}$ stands for interaction with the bath. We define $G_{1}$ as follows. There is an exponential clock at each edge $e=(u, v) \in \mathcal{E}\left(\mathcal{D}_{L}\right)$ of rate $e_{(u, v)}$. When it rings, the energies of the 
two corresponding systems $\left(\xi_{u}\right.$ and $\left.\xi_{v}\right)$ are pooled together and redistributed according to a $\operatorname{Beta}\left(\omega_{u} / 2, \omega_{v} / 2\right)$ distribution. Thus

$$
\left(G_{1} f\right)(\underline{\xi})=\sum_{(u, v) \in \mathcal{E}\left(\mathcal{D}_{L}\right)} r_{(u, v)} \int_{0}^{1} \frac{1}{B\left(\frac{\omega_{u}}{2}, \frac{\omega_{v}}{2}\right)} p^{\frac{\omega_{u}}{2}-1}(1-p)^{\frac{\omega_{v}}{2}-1}\left[f\left(\underline{\xi^{\prime}}\right)-f(\underline{\xi})\right] d p
$$

where

$$
\xi_{w}^{\prime}=\left\{\begin{aligned}
\xi_{w} & \text { if } w \notin\{u, v\} \\
p\left(\xi_{u}+\xi_{v}\right) & \text { if } w=u \\
(1-p)\left(\xi_{u}+\xi_{v}\right) & \text { if } w=v
\end{aligned}\right.
$$

Every edge $e=(u, v)$ where $u \in \mathcal{D}_{L}$ and $v \in \mathbb{Z}^{d} \backslash \mathcal{D}_{L}$ provides connection to the heat bath of temperature $T(v / L)$ : with rate $r_{(u, v)}, \xi_{u}$ is updated to $\Gamma\left(\frac{\omega_{u}}{2}, T\left(\frac{v}{L}\right)\right)$. That is,

$$
\left(G_{2} f\right)(\underline{\xi})=\sum_{u \in \mathcal{D}_{L}, v \in \mathbb{Z}^{d} \backslash \mathcal{D}_{L},\|u-v\|=1} r_{(u, v)} \int_{0}^{\infty} \frac{\eta^{\frac{\omega_{u}}{2}-1} \exp \left[-\frac{\eta}{T\left(\frac{v}{L}\right)}\right]}{\left[T\left(\frac{v}{L}\right)\right]^{\frac{\omega_{u}}{2}} \Gamma\left(\frac{\omega_{u}}{2}\right)}\left[f\left(\underline{\xi}^{\prime \prime}\right)-f(\underline{\xi})\right] d \eta
$$

where

$$
\xi_{w}^{\prime \prime}=\left\{\begin{aligned}
\xi_{w} & \text { if } w \neq u \\
\eta & \text { if } w=u
\end{aligned}\right.
$$

This completes the definition of $\boldsymbol{X}^{(L)}(t)$.

\section{THE DUAL PROCESS}

As mentioned in the Introduction, we want to understand the asymptotic behavior of $\boldsymbol{X}(t)$ by switching to a dual process. This section is devoted to the discussion of duality.

For $\mathcal{D} \subset \mathbb{R}^{d}$ and $L$ as in Section 2, we now introduce a Markov process $\boldsymbol{Y}^{(L)}(t)=\left(\left(n_{v}^{(L)}(t)\right)_{v \in \mathcal{D}_{L}},\left(\hat{n}_{v}^{(L)}(t)\right)_{v \in \mathcal{B}_{L}}\right)$ designed to carry certain dual object, which we call particles, from sites in $\mathcal{D}_{L}$ to

$$
\mathcal{B}_{L}=\left\{v \in \mathbb{Z}^{d} \backslash \mathcal{D}_{L}: \exists v \in \mathcal{D}_{L}:\|u-v\|=1\right\} .
$$

Here, $n_{v}$ the number of particles at site $v \in \mathcal{D}_{L}$ and $\hat{n}_{w}$ the number of particles permanently drooped off to the storage at $w \in \mathcal{B}_{L}$. The generator of the process $\boldsymbol{Y}(t)$ is given by

$$
(A f)(\underline{n})=\left(A_{1} f\right)(\underline{n})+\left(A_{2} f\right)(\underline{n}),
$$

where $A_{1}$ corresponds to movements inside $\mathcal{D}_{L}$ and $A_{2}$ corresponds to the process of dropping off the particles to the storage. That is,

$$
\begin{aligned}
& \left(A_{1} f\right)(\underline{n})=\sum_{(u, v) \in \mathcal{E}\left(\mathcal{D}_{L}\right)} r_{(u, v)} \\
& \sum_{k=0}^{n_{v}+n_{w}}\left(\begin{array}{c}
n_{u}+n_{v} \\
k
\end{array}\right) \frac{B\left(k+\frac{\omega_{u}}{2}, n_{u}+n_{v}-k+\frac{\omega_{v}}{2}\right)}{B\left(\frac{\omega_{u}}{2}, \frac{\omega_{v}}{2}\right)}\left[f\left(\underline{n}^{\prime}\right)-f(\underline{n})\right]
\end{aligned}
$$


where

$$
n_{w}^{\prime}=\left\{\begin{aligned}
n_{w} & \text { if } w \notin\{u, v\} \\
k & \text { if } w=u . \\
n_{v}+n_{w}-k & \text { if } w=v .
\end{aligned} \quad \text { and } \quad \hat{n}_{w}^{\prime}=\hat{n}_{w} \forall w \in \mathcal{B}_{L}\right.
$$

Recall that in case of the process $\boldsymbol{X}$, the energies are redistributed according to a beta distribution with parameters $\omega_{u} / 2, \omega_{v} / 2$. In case of the dual process $\boldsymbol{Y}$, we redistribute the particles with the so called beta binomial distribution: first we choose a $p$ according to $\operatorname{Beta}\left(\omega_{u} / 2, \omega_{v} / 2\right)$, then we choose $n_{u}^{\prime}$ with binomial distribution of parameters $n_{u}+n_{v}, p$ and $n_{v}^{\prime}=n_{u}+n_{v}-n_{u}^{\prime}$.

The second part of the generator is given by

$$
\left(A_{2} f\right)(\underline{n})=\sum_{u \in \mathcal{D}_{L}, v \in \mathcal{B}_{L},\|u-v\|=1} r_{(u, v)}\left[f\left(\underline{n}^{\prime}\right)-f(\underline{n})\right]
$$

where

$$
n_{w}^{\prime}=\left\{\begin{aligned}
n_{w} & \text { if } w \neq u \\
0 & \text { if } w=u
\end{aligned} \quad \text { and } \quad \hat{n}_{w}^{\prime}=\left\{\begin{aligned}
\hat{n}_{w} & \text { if } w \neq v \\
\hat{n}_{v}+n_{u} & \text { if } w=v
\end{aligned}\right.\right.
$$

This completes the definition of $\boldsymbol{Y}^{(L)}(t)$.

Now we turn to the duality. Let us define the function with respect to which the duality holds

$$
F(\underline{n}, \underline{\xi})=\prod_{u \in \mathcal{D}_{L}} \frac{\xi_{u}^{n_{u}} \Gamma\left(\omega_{u} / 2\right)}{\Gamma\left(n_{u}+\omega_{u} / 2\right)} \prod_{v \in \mathcal{B}_{L}}\left[T\left(\frac{v}{L}\right)\right]^{\hat{n}_{v}}
$$

The duality with respect to $F$ means that

Proposition 1. For any $\underline{\xi}$ any $\underline{n}$ and any $t>0$,

$$
\mathbb{E}\left(F(\underline{n}, \boldsymbol{X}(t)) \mid \boldsymbol{X}_{0}=\underline{\xi}\right)=\mathbb{E}\left(F(\boldsymbol{Y}(t), \underline{\xi}) \mid \boldsymbol{Y}_{0}=\underline{\xi}\right)
$$

Proof. Clearly it is enough to prove that for any $\underline{\xi}$ and $\underline{n}$,

$$
G F(\underline{\xi}, \underline{n})=A F(\underline{\xi}, \underline{n}) \text {. }
$$

To prove this, we consider the following two cases, where Case $i$ corresponds to $G_{i}$ and $A_{i}$ for $i=1,2$.

Case 1. The clock on the edge $(u, v)$ rings. The term corresponding to $u$ and $v$ in $G_{1} F$ can be written as $r_{(u, v)} \cdot I \cdot I I$, where

$$
I=\Gamma\left(\omega_{u} / 2\right) \Gamma\left(\omega_{v} / 2\right) \prod_{w \in \mathcal{D}_{L} \backslash\{u, v\}} \frac{\xi_{w}^{n_{w}} \Gamma\left(\omega_{w} / 2\right)}{\Gamma\left(n_{w}+\omega_{w} / 2\right)} \prod_{w \in \mathcal{B}_{L}}\left[T\left(\frac{w}{L}\right)\right]^{\hat{n}_{w}}
$$

and

$$
I I=\frac{\int_{0}^{1} p^{n_{u}+\frac{\omega_{u}}{2}-1}(1-p)^{n_{v}+\frac{\omega_{v}}{2}-1} \frac{1}{B\left(\frac{\omega_{u}}{2}, \frac{\omega_{v}}{2}\right)}\left(\xi_{u}+\xi_{v}\right)^{n_{u}+n_{v}} d p-\xi_{u}^{n_{u}} \xi_{v}^{n_{v}}}{\Gamma\left(n_{u}+\frac{\omega_{u}}{2}\right) \Gamma\left(n_{v}+\frac{\omega_{v}}{2}\right)}
$$


Then we compute

$$
\begin{aligned}
& I I+\frac{\xi_{u}^{n_{u}} \xi_{v}^{n_{v}}}{\Gamma\left(n_{u}+\frac{\omega_{u}}{2}\right) \Gamma\left(n_{v}+\frac{\omega_{v}}{2}\right)}= \\
= & \frac{1}{B\left(\frac{\omega_{u}}{2}, \frac{\omega_{v}}{2}\right)} \frac{1}{\Gamma\left(n_{u}+n_{v}+\frac{\omega_{u}+\omega_{v}}{2}\right)}\left(\xi_{u}+\xi_{v}\right)^{n_{u}+n_{v}} \\
= & \frac{1}{B\left(\frac{\omega_{u}}{2}, \frac{\omega_{v}}{2}\right)} \frac{1}{\Gamma\left(n_{u}+n_{v}+\frac{\omega_{u}+\omega_{v}}{2}\right)} \sum_{k=0}^{n_{u}+n_{v}}\left(\begin{array}{c}
n_{u}+n_{v} \\
k
\end{array}\right) \xi_{u}^{n_{u}} \xi_{v}^{n_{v}} \\
= & \frac{1}{B\left(\frac{\omega_{u}}{2}, \frac{\omega_{v}}{2}\right)} \sum_{k=0}^{n_{u}+n_{v}}\left(\begin{array}{c}
n_{u}+n_{v} \\
k
\end{array}\right) B\left(k+\frac{\omega_{u}}{2}, n_{u}+n_{v}-k+\frac{\omega_{v}}{2}\right) \frac{\xi_{u}^{n_{u}}}{\Gamma\left(k+\frac{\omega_{u}}{2}\right)} \frac{\xi_{v}^{n_{v}}}{\Gamma\left(n-k+\frac{\omega_{v}}{2}\right)}
\end{aligned}
$$

Thus $r_{(u, v)} \cdot I \cdot I I$ is the term corresponding to $u$ and $v$ in $A_{1} F$.

Case 2. The energy at site $u \in \mathcal{D}_{L}$ is updated by the heat bath at $v \in \mathcal{B}_{L}$ (where $\|u-v\|=1$ ). As before, we write the term corresponding to $(u, v)$ in $G_{2} F$ as $r_{(u, v)} \cdot I \cdot I I$, where

$$
I=\prod_{u^{\prime} \in \mathcal{D}_{L} \backslash\{u\}} \frac{\xi_{u^{\prime}}^{n_{u^{\prime}}} \Gamma\left(\omega_{u^{\prime}} / 2\right)}{\Gamma\left(n_{u^{\prime}}+\omega_{u^{\prime}} / 2\right)} \prod_{v^{\prime} \in \mathcal{B}_{L} \backslash\{v\}}\left[T\left(\frac{v^{\prime}}{L}\right)\right]^{\hat{n}_{v^{\prime}}}
$$

and

$$
I I=\frac{\Gamma\left(\frac{\omega_{u}}{2}\right)}{\Gamma\left(n_{u}+\frac{\omega_{u}}{2}\right)}\left[\int_{0}^{\infty} \frac{\eta^{n_{u}+\frac{\omega_{u}}{2}-1} \exp \left[-\frac{\eta}{T\left(\frac{v}{L}\right)}\right]}{\left[T\left(\frac{v}{L}\right)\right]^{\frac{\omega_{u}}{2}} \Gamma\left(\frac{\omega_{u}}{2}\right)} d \eta-\xi_{u}^{n_{u}}\right]\left[T\left(\frac{v}{L}\right)\right]^{\hat{n}_{v}}
$$

By (1), we obtain that

$$
I I+\frac{\xi_{u}^{n_{u}} \Gamma\left(\frac{\omega_{u}}{2}\right)}{\Gamma\left(n_{u}+\frac{\omega_{u}}{2}\right)}\left[T\left(\frac{v}{L}\right)\right]^{\hat{n}_{v}}=\left[T\left(\frac{v}{L}\right)\right]^{\hat{n}_{v}+n_{u}}
$$

Thus $r_{(u, v)} \cdot I \cdot I I$ is the term corresponding to $v$ in $A_{2} F$.

Note that the process $\boldsymbol{Y}$ preserves the total number of particles, which will be denoted be $N$. We conclude this section with the following simple lemma.

Lemma 1. The restriction of the process $\boldsymbol{Y}$ to arbitrary subset of $K$ particles (with $K<N)$ is also a Markov process and satisfies the definition of $\boldsymbol{Y}$ with $N$ replaced by $K$.

Proof. Without loss of generality, we can consider a system with $K=N-1$ particles, assume that the clock attached to the edge $(v, w)$ rings and the union of the particles at sites $u$ and $v$ prior to the mixing are labeled $\{1, \ldots, n\}$. 
Then the probability that after the mixing, the set of particles at site $u$ is exactly $\left\{j_{1}, \ldots j_{l}\right\} \subset\{1,2, \ldots, n\}$ is given by

$$
p\left(j_{1}, \ldots, j_{l}\right)=\left(\begin{array}{l}
n \\
l
\end{array}\right) \frac{\Gamma\left(l+\omega_{u} / 2\right) \Gamma\left(n-l+\omega_{v} / 2\right)}{\Gamma\left(n+\omega_{u} / 2+\omega_{v} / 2\right)} \frac{\Gamma\left(\omega_{u} / 2+\omega_{v} / 2\right)}{\Gamma\left(\omega_{u} / 2\right) \Gamma\left(\omega_{v} / 2\right)} \frac{1}{\left(\begin{array}{l}
n \\
l
\end{array}\right)}
$$

Now assume we add a new particle (of index $N$ ). If this new particle is not at sites $u$ or $v$, then clearly the situation is not disturbed. If it is there, we compute

$$
\begin{aligned}
& p\left(j_{1}, \ldots, j_{l}\right)+p\left(j_{1}, \ldots, j_{l}, N+1\right)= \\
& \frac{\Gamma\left(l+\omega_{u} / 2\right) \Gamma\left(n+1-l+\omega_{v} / 2\right)}{\Gamma\left(n+1+\omega_{u} / 2+\omega_{v} / 2\right)} \frac{\Gamma\left(\omega_{u} / 2+\omega_{v} / 2\right)}{\Gamma\left(\omega_{u} / 2\right) \Gamma\left(\omega_{v} / 2\right)} \\
& +\frac{\Gamma\left(l+1+\omega_{u} / 2\right) \Gamma\left(n-l+\omega_{v} / 2\right)}{\Gamma\left(n+1+\omega_{u} / 2+\omega_{v} / 2\right)} \frac{\Gamma\left(\omega_{u} / 2+\omega_{v} / 2\right)}{\Gamma\left(\omega_{u} / 2\right) \Gamma\left(\omega_{v} / 2\right)}
\end{aligned}
$$

An elementary computation shows that (5) is equal to (66). The lemma follows.

\section{Local Equilibrium}

Let $d, \mathcal{D}$ and $L$ be as before such that $\mathcal{D}_{L}$ is connected.

First we state the existence and uniqueness of invariant measure in the equilibrium case.

Proposition 2. Fix arbitrary functions $\omega: \mathcal{D}_{L} \rightarrow \mathbb{Z}_{+}$and $r: \mathcal{E}\left(\mathcal{D}_{L}\right) \rightarrow \mathbb{R}_{+}$. If $T$ is constant, then

$$
\mu_{e}^{(L)}=\prod_{v \in \mathcal{D}_{L}} \Gamma\left(\frac{\omega_{v}}{2}, \frac{1}{T}\right)
$$

is the unique invariant probability measure of the process $\boldsymbol{X}^{(L)}(t)$.

Proof. The discussion in Section 1.1 implies the following statement (which is actually well known, see e.g. Lemma 3 in [CHS81]). Let $\xi_{1}$ and $\xi_{2}$ be independent Gamma distributed random variables with shape parameter $k$ and $l$, respectively and with the same scale parameter. Let $Z$ be independent from $X$ and $Y$ and have Beta distribution with parameters $k / 2$ and $l / 2$. Then the pair $\left(Z\left(\xi_{1}+\xi_{2}\right),(1-Z)\left(\xi_{1}+\xi_{2}\right)\right)$ has the same distribution as $\left(\xi_{1}, \xi_{2}\right)$. Proposition 2 follows.

Our primary interest is in the out-of-equilibrium settings where the bath temperature is non constant:

Proposition 3. Let $\omega: \mathcal{D}_{L} \rightarrow \mathbb{Z}_{+}$and $r: \mathcal{E}\left(\mathcal{D}_{L}\right) \rightarrow \mathbb{R}_{+}$be arbitrary. The process $\boldsymbol{X}^{(L)}(t)$ has a unique invariant probability measure $\mu^{(L)}$. Furthermore, the distribution of $\boldsymbol{X}^{(L)}(t)$ converges to $\mu^{(L)}$ as $t \rightarrow \infty$ for any initial distribution of $\boldsymbol{X}^{(L)}(0)$. 
We skip the proof of Proposition 3 since it is very similar to the analogous propositions in earlier similar models, see Proposition 1.2 in [RY07], Proposition 2 in [LNY15].

4.1. Hydrodynamic limit. In order to discuss the local equilibrium in the hydrodynamic limit, we need some definitions. First we introduce some properties of the initial measures.

Definition 1. We say that $\boldsymbol{X}(0)$ is associated with $f$ if for any fixed $\delta$ and any $k$

$$
\mathbb{E}\left(\prod_{i=1}^{k} \xi_{v_{i}}^{(L)}(0)\right) \sim \prod_{i=1}^{k} \frac{\omega_{v_{i}}}{2} f\left(v_{i} / L\right)
$$

as $L \rightarrow \infty$ uniformly for every $v_{1}, \ldots, v_{k} \in \mathcal{D}_{L}$ satisfying $\left\|v_{i}-v_{j}\right\| \geq \delta L$ for $i \neq j$ and with some fixed continuous function $f: \mathbb{R}^{d} \rightarrow \mathbb{R}_{+}$such that $\left.f\right|_{\mathbb{R}^{d} \backslash D}=T$

Definition 2. We say that $\boldsymbol{X}(0)$ satisfies the uniform moment condition if there are constants $C_{k}$ such that $\mathbb{E}\left(\xi_{v}^{k}(0)\right)<C_{k}$ for every $L$ and for every $v \in \mathcal{D}_{L}$.

Recall that the Lévy-Prokhorov distance is the metrization of weak convergence of measures.

Definition 3. We say that $\boldsymbol{X}^{(L)}(t)$ approaches local equilibrium in the hydrodynamic limit at $x \in \mathcal{D}$ and $t>0$ if for any finite set $S \subset \mathbb{Z}^{d}$ the LévyProkhorov distance of the distribution of $\boldsymbol{X}^{(L)}\left(t L^{2}\right)$ restricted to the components $\left(\xi_{\langle x L\rangle+s}\right)_{s \in S}$ and

$$
\prod_{s \in S} \Gamma\left(\omega_{\langle x L\rangle+s} / 2, u(t, x)\right)
$$

converges to zero as $L \rightarrow \infty$.

We will choose the initial distributions, i.e. the distributions of $\boldsymbol{X}^{(L)}(0)$, which are associated with a continuous function $f$. The interesting question is that what kind of equation defines $u$ for different choices of $\omega$ and $r$. In any case, we expect that the initial condition is given by $f$ and the boundary condition by $T$. We will consider the two simplest cases here.

Theorem 1. Assume $d \geq 2$ and $\omega_{v}=\omega_{0} \in \mathbb{Z}_{+}$for every site $v$. Assume furthermore that $r_{(u, v)}=R\left(\frac{u+v}{2 L}\right)$ for every $u \in \mathcal{D}_{L}$ and $v \in \mathbb{Z}^{d}$ with $\|u-v\|=1$, where $R \in \mathcal{C}^{2}\left(\mathbb{R}^{d}, \mathbb{R}_{+}\right)$. Also assume that $\boldsymbol{X}^{(L)}(0)$ is associated with $f$, a continuous function $f: \overline{\mathcal{D}} \rightarrow \mathbb{R}_{+}$, and satisfy the uniform moment condition. Then $\boldsymbol{X}^{(L)}(t)$ approaches local equilibrium in the hydrodynamic limit for all $x \in \mathcal{D}$ and $t>0$ with $u$ the unique solution of the equation

$$
\left\{\begin{array}{l}
u_{t}=\nabla(R \nabla u), \\
u(0, x)=f(x), \\
\left.u(t, x)\right|_{\partial \mathcal{D}}=T(x) .
\end{array}\right.
$$


Remark about the initial conditions Note that $f$ represents the energy per degrees of freedom at time zero, that is why we need the multiplier $\omega_{v} / 2$ on the right hand side of (7). We do not have to assume local equilibrium at zero, which would correspond to the special choice of $\boldsymbol{X}(0)$ : the product of Gamma distributions (with shape parameter $\omega_{v} / 2$, and scale parameter $f(v / L))$. One interesting consequence of Theorem [1 (and similarly that of Theorem 2) is that the system satisfies the local equilibrium for arbitrary positive macroscopic time even if it only satisfies the given weaker condition at time zero. Since we want to leverage the duality via moments, we also need to assume some condition on the higher moments. The simplest one is the uniform moment condition. Most probably, neither condition (77) nor the uniform moment condition is optimal, but we do not pursue the most general case here.

Our next choice is the simplest non-continuous environment: we consider $\mathcal{D}=[-1,1]^{d}$ with one of the functions $\omega$ and $r$ being constant on $\mathcal{D}$ and the other one is constant on $[-1,0] \times[-1,1]^{d-1}$ and $[0,1] \times[-1,1]^{d-1}$. Thus we have the following

Theorem 2. Let $d \geq 2, \mathcal{D}=[-1,1]^{d}$. Assume that $\boldsymbol{X}^{(L)}(0)$ is associated with $f$, a continuous extension of $T$ to $\overline{\mathcal{D}}$, and satisfies the uniform moment condition

(a) Let $\omega_{v}=\omega_{-1}$ if $v_{1}<0$ and $\omega_{v}=\omega_{1}$ if $v_{1} \geq 0$ with some positive integers $\omega_{-1}, \omega_{1}$ and let $r$ be constant. Then $\overline{\boldsymbol{X}}^{(L)}(t)$ approaches local equilibrium in the hydrodynamic limit for all $x \in \mathcal{D}$ with $x_{1} \neq 0$ and all $t>0$ with $u$ the unique solution of the equation

$$
\left\{\begin{array}{l}
u_{t}=r \Delta u \quad \text { for } x \in \mathcal{D} \text { with } x_{1} \neq 0 \\
\omega_{1} \frac{\partial}{\partial x_{1}+} u\left(t,\left(0, x_{2}, \ldots, x_{d}\right)\right)=\omega_{-1} \frac{\partial}{\partial x_{1}-} u\left(t,\left(0, x_{2}, \ldots, x_{d}\right)\right) \\
u(0, x)=f(x) \\
\left.u(t, x)\right|_{\partial \mathcal{D}}=T(x) .
\end{array}\right.
$$

(b) Let $r_{(u, v)}=r_{-1}$ if $u_{1}+v_{1}<0$ and $r_{(u, v)}=r_{1}$ otherwise, where $r_{-1}, r_{1}$ are fixed positive numbers. Similarly, $r_{w}=r_{-1}$ if $w_{1}<0$ and $r_{w}=r_{1}$ otherwise. Let $\omega$ be constant. Then $\boldsymbol{X}^{(L)}(t)$ approaches local equilibrium in the hydrodynamic limit for all $x \in \mathcal{D}$ with $x_{1} \neq 0$ and all $t>0$ with $u$ the unique solution of the equation

$$
\left\{\begin{array}{l}
u_{t}=r_{\operatorname{sign}\left(x_{1}\right)} \Delta u \quad \text { for } x \in \mathcal{D} \text { with } x_{1} \neq 0, \\
r_{1} \frac{\partial}{\partial x_{1}+} u\left(t,\left(0, x_{2}, \ldots, x_{d}\right)\right)=r_{-1} \frac{\partial}{\partial x_{1}-} u\left(t,\left(0, x_{2}, \ldots, x_{d}\right)\right) \\
u(0, x)=f(x), \\
\left.u(t, x)\right|_{\partial \mathcal{D}}=T(x) .
\end{array}\right.
$$

4.2. Nonequilibrium steady state. Now we are interested in the invariant measure of the Markov chains for finite (but large) $L$. Specifically, we are looking for a function $u(x), x \in \mathcal{D}$ such that in the $\operatorname{limit}_{\lim _{L \rightarrow \infty}} \lim _{t \rightarrow \infty}$ the 
local temperature exists and is given by $u(x)$. In case the hydrodynamic limit is known, $u(x)$ is expected to be equal to $\lim _{t \rightarrow \infty} u(t, x)$. We will choose arbitrary environment $(r$ and $\omega)$, that is why we define the local equilibrium in a little more general form, namely with an $L$ dependent $u$. Of course in all natural examples, one expects $u^{(L)}$ to converge.

Definition 4. We say that $\boldsymbol{X}^{(L)}(t)$ approaches local equilibrium in the nonequilibrium steady state if for any $x \in \mathcal{D}$ and any finite set $S \subset \mathbb{Z}^{d}$ the LévyProkhorov distance of the invariant measure of $\boldsymbol{X}^{(L)}(t)$ restricted to the components $\left(\xi_{\langle x L\rangle+s}\right)_{s \in S}$ and

$$
\prod_{s \in S} \Gamma\left(\omega_{\langle x L\rangle+s} / 2, u^{(L)}(x)\right)
$$

converges to zero as $L \rightarrow \infty$.

Let us fix $d=1$ and $\mathcal{D}=(0,1)$. To simplify notation, we will write $r_{m+1 / 2}:=$ $r_{(m, m+1)}, \omega_{0}:=\omega_{1}$ and $\omega_{L}:=\omega_{L-1}$. Furthermore, we will need the following two definitions:

$$
\psi(m)=\frac{\omega_{m-1}+\omega_{m}}{r_{m-1 / 2} \omega_{m-1} \omega_{m}} \quad \text { for } 1 \leq m \leq L
$$

and

$$
\mathcal{A}^{(L)}(x)=\frac{\sum_{m=1}^{\lfloor x L\rfloor} \psi(m)}{\sum_{m=1}^{L} \psi(m)} .
$$

Theorem 3. Let $d=1$ and $\mathcal{D}=(0,1)$. Assume that the functions $r$ and $\omega$ are bounded away from zero and infinity uniformly in $L$ and the temperature on the boundary is given by $T(0), T(1) \in \mathbb{R}_{+} \cup\{0\}$. Then $\boldsymbol{X}^{(L)}(t)$ approaches local equilibrium in the nonequilibrium steady state with

$$
u^{(L)}(x)=\left(1-\mathcal{A}^{(L)}(x)\right) T(0)+\mathcal{A}^{(L)}(x) T(1) .
$$

Clearly, $u^{(L)}(x)$ can easily diverge in this generality. That is why we consider two special cases. In case (a), $r$ is constant and $\omega$ is random. In this case, we prove the quenched local equilibrium in the nonequilibrium steady state, i.e. the almost sure convergence of $u^{(L)}(x)$ to a deterministic limit. In case (b), $\omega$ is constant but $r$ is prescribed by a non-constant macroscopic function. We also prove that $u^{(L)}(x)$ converges in this case.

Proposition 4. (a) Let $r$ be constant, $K$ be the maximal degrees of freedom and let us fix some continuous function

$$
\varkappa:[0,1] \rightarrow\left\{p \in \mathbb{R}^{K}: p \geq 0, \sum_{i=1}^{K} p_{i}=1\right\} .
$$

For each L, let us choose $\omega_{v}^{(L)}$ randomly and independently from one another with

$$
\mathbb{P}\left(\omega_{v}^{(L)}=i\right)=\varkappa_{i}(v / L)
$$


Then for almost every realization of the random functions $\omega^{(L)}$,

$$
\lim _{L \rightarrow \infty} \mathcal{A}^{(L)}(x)=\frac{\sum_{i=1}^{K} \frac{1}{i} \int_{0}^{x} \varkappa_{i}(y) d y}{\sum_{i=1}^{K} \frac{1}{i} \int_{0}^{1} \varkappa_{i}(y) d y} .
$$

(b) Let $\omega$ be constant, $\varrho:[0,1] \rightarrow \mathbb{R}_{+}$is a continuous function. For each $L$ and $v=0,1, \ldots L-1$ define $r_{v+1 / 2}^{(L)}=\varrho(v / L)$. Then

$$
\lim _{L \rightarrow \infty} \mathcal{A}^{(L)}(x)=\frac{\int_{0}^{x} 1 / \varrho(y) d y}{\int_{0}^{1} 1 / \varrho(y) d y} .
$$

Before turning to the proofs of the above results, we briefly comment on some possibilities of extension.

4.3. Possible extensions. As we will see, the proof of Theorems 1 and 2 also provides the local equilibrium in the nonequilibrium steady state.

Corollary 4. Consider the setup of either Theorem 1 or 2 . Then $\boldsymbol{X}^{(L)}(t)$ approaches local equilibrium (assuming $x_{1} \neq 0$ in case of Theorem Q) in the nonequilibrium steady state with $U(x)=\lim _{t \rightarrow \infty} u(t, x)$.

Indeed, for $\delta>0$ fixed one can find some $t$ large such that with probability at least $1-\delta$, all processes in Proposition 6 have arrived at the boundary before $t L^{2}$. In such cases, $\tilde{\boldsymbol{Y}}_{i}^{(L)}\left(t L^{2}\right)=\tilde{\boldsymbol{Y}}_{i}^{(L)}(\infty)$, and the latter can be used to prove local equilibrium in the nonequilibrium steady state (cf. the proof of Theorem 3).

Furthermore, it seems likely that our proof could be adapted to a version of Theorem 2 with more general domains and with piecewise constant $r$ and $\omega$ (see [P90] for the extension of skew Brownian motion to such scenarios).

However, the case of more general inhomogeneity in either high dimensions or in the hydrodynamic limit can be difficult. For example, if $\varkappa$ is constant in Proposition 4(a), then in order to verify the hydrodynamic limit, one would have to compute the scaling limit of some interacting random walkers among iid conductances, whereas even the case of one random walker is non obvious (see [SSz04]). Clearly, the case of high dimensions or non iid environments are even much harder.

\section{Proof of Theorems 1 and 2}

Since the proofs of Theorems 1 and 2 are very similar, we provide one proof and distinguish between cases Theorems 1, 2(a) and 2(b) if necessary. Let us fix some $t>0$, a point $x \in \mathcal{D}$ and a finite set $S \subset \mathbb{Z}^{d}$. We need to show that the (joint) distribution of $\left(\xi_{\langle x L\rangle+s}\left(t L^{2}\right)\right)_{s \in S}$ converges to the product of gamma distributions with the scale parameter $u(t, x)$. As it is well known, any product of Gamma distributions is characterized by its moments (see [Z83]). Thus it is enough to prove that the moments of $\left(\xi_{\langle x L\rangle+s}\right)_{s \in S}$ converge to the product of the moments of gamma distributions (convergence of second moments implies 
tightness). When computing the moments of order $n_{s}^{*} \in \mathbb{N}, s \in S$, we can use Proposition 1 to switch to the dual process.

We need to introduce some auxiliary processes. Let us denote by $\tilde{\boldsymbol{Y}}$ the slight variant of $\boldsymbol{Y}$ where the position of distinguishable particles are recorded. More precisely, the phase space of $\tilde{\boldsymbol{Y}}=\left(\tilde{\boldsymbol{Y}}_{i}^{(L)}(t)\right)_{0 \leq t, 1 \leq i \leq N}$ is $\left(\mathcal{D}_{L} \cup \mathcal{B}_{L}\right)^{N}$, where $N=\sum_{s \in S} n_{s}^{*}$ and the initial condition is given such that for all $s \in S$,

$$
\#\left\{i: \tilde{\boldsymbol{Y}}_{i}(0)=\langle x L\rangle+s\right\}=n_{s}^{*} .
$$

For any $t \geq 0$ we define $\tilde{\boldsymbol{Y}}(t)$ so that

$$
\#\left\{i: \tilde{\boldsymbol{Y}}_{i}(t)=v \in \mathcal{D}_{L}\right\}=n_{v}\left(t_{k}\right), \quad \#\left\{i: \tilde{\boldsymbol{Y}}_{i}(t)=v \in \mathcal{B}_{L}\right\}=\hat{n}_{v}\left(t_{k}\right)
$$

and for all $t$,

$$
\#\left\{i: \tilde{\boldsymbol{Y}}_{i}(t-) \neq \tilde{\boldsymbol{Y}}_{i}(t+)\right\} \leq 1 .
$$

We want to show that the diffusively rescaled version of $\tilde{\boldsymbol{Y}}$ converges weakly to $N$ independent copies of some (generalized) diffusion processes $\mathcal{Y}$. Then the Kolmogorov backward equation associated with these processes will provide the function $u$. Clearly, the process $\mathcal{Y}$ will have to be stopped on $\partial \mathcal{D}$. So as to shed light on the main component of the proof, namely the convergence to the diffusion process, we introduce a further simplification by not stopping the particles.

We can assume that $R$ is bounded in case of Theorem 1 (possibly by multiplying with a smooth function which is constant on $\mathcal{D}$ and decays quickly) and extend the definition of $\omega_{v}, r_{(u, v)}$ in case of Theorem 2 for any $u, v \in \mathbb{Z}^{d}$. Then we consider the process $\boldsymbol{Z}(t)=\left(n_{v}(t)\right)_{v \in \mathbb{Z}^{d}}$ on the space

$$
z_{v} \in \mathbb{Z}_{+}, \quad \sum_{v \in \mathbb{Z}^{d}} z_{v}=N
$$

with the generator $A_{1}^{\prime}$, obtained from $A_{1}$ by replacing $\sum_{(u, v) \in \mathcal{E}\left(\mathcal{D}_{L}\right)}$ with $\sum_{(u, v) \in \mathcal{E}\left(\mathbb{Z}^{d}\right)}$. Then we define $\tilde{\boldsymbol{Z}}$ from $\boldsymbol{Z}$ the same way as we defined $\tilde{\boldsymbol{Y}}$ from $\boldsymbol{Y}$. Observe that by construction (and with the natural coupling) for $N=1$ we have

$$
\tilde{\boldsymbol{Y}}^{(L)}(s)=\tilde{\boldsymbol{Z}}\left(s \wedge \tau_{\mathcal{D}_{L}}\right) \quad \text { where } \quad \tau_{\mathcal{D}_{L}}=\min \left\{s: \tilde{\boldsymbol{Z}}(s) \notin \mathcal{D}_{L}\right\}
$$

Now we define the limiting process $(\mathcal{Z}(s))_{0 \leq s \leq t}$ with $\mathcal{Z}(0)=x$ and with the generator

$$
\mathcal{L}= \begin{cases}\sum_{i=1}^{d} R(x) \frac{\partial^{2}}{\partial x_{i}^{2}}+\sum_{i=1}^{d} \frac{\partial R}{\partial x_{i}} \frac{\partial}{\partial x_{i}} & \text { in Theorem 1 } \\ \sum_{i=1}^{d} r \frac{\partial^{2}}{\partial x_{i}^{2}} & \text { in Theorem 2(a) } \\ \sum_{i=1}^{d} r_{\operatorname{sign}\left(x_{1}\right) \frac{\partial^{2}}{\partial x_{i}^{2}}} & \text { in Theorem 2(b). }\end{cases}
$$

acting on

(1) functions $\phi \in \mathcal{C}_{0}^{2}$ in case of Theorem 1 
(2) compactly supported continuous functions $\phi$ which admit $\mathcal{C}^{2}$ extensions on $\left(\mathbb{R}_{-} \cup 0\right) \times \mathbb{R}^{d-1}$ and $\left(\mathbb{R}_{+} \cup 0\right) \times \mathbb{R}^{d-1}$ and

$$
\begin{cases}\omega_{-1} \frac{\partial}{\partial x_{1}-} \phi\left(0, x_{2}, \ldots, x_{d}\right)=\omega_{1} \frac{\partial}{\partial x_{1}+} \phi\left(0, x_{2}, \ldots, x_{d}\right) & \text { in case of Theorem 2(a) } \\ r_{-1} \frac{\partial}{\partial x_{1}-} \phi\left(0, x_{2}, \ldots, x_{d}\right)=r_{1} \frac{\partial}{\partial x_{1}+} \phi\left(0, x_{2}, \ldots, x_{d}\right) & \text { in case of Theorem 2(b) }\end{cases}
$$

Note that $\mathcal{Z}$ is a diffusion process in case of Theorem 1 and a generalized diffusion process in case of Theorem 2 (see [P90] for a survey on generalized diffusion processes and A78 for an early proof of the existence of $\mathcal{Z}$ in case of Theorem $2(\mathrm{~b}))$. Now let us stop $\mathcal{Z}$ on $\partial \mathcal{D}$ and define

$$
\mathcal{Y}(s)=\mathcal{Z}\left(s \wedge \tau_{\mathcal{D}}\right) \quad \text { where } \quad \tau_{\mathcal{D}}=\min \{s: \mathcal{Z}(s) \in \partial \mathcal{D}\}
$$

The connection between the processes $\tilde{\boldsymbol{Y}}$ and the PDE's defining $u$ is most easily seen in the simplest case of one particle.

Let $\Rightarrow$ denote weak convergence in the Skorokhod space $\mathcal{D}[0, T]$ with respect to the supremum metric and with some $T$ to be specified. (Although we need the Skorokhod space as the trajectories of $\tilde{Z}$ are not continuous, but the limiting measures will always be supported on $\mathcal{C}[0, T]$ and we can use the supremum metric. Alternatively, one could smooth the trajectories of $\tilde{Z}$ and only use the space $\mathcal{C}[0, T]$.)

Lemma 2. If $N=1$, then

$$
\left(\frac{\tilde{\boldsymbol{Z}}\left(s L^{2}\right)}{L}\right)_{0 \leq s \leq t} \Rightarrow(\mathcal{Z}(s))_{0 \leq s \leq t}
$$

Proof. In the setup of Theorem 1, this follows from Theorem 11.2.3 in [SV07]. More precisely, as we can neglect events of small probability, we can assume that (A) the particle jumps less than $L^{3}$ times before $t L^{2}$ and consequently (B) the smallest time between two consecutive jumps before $t L^{2}$ is bigger than $L^{-4}$. Now choosing $h=L^{-4}, \tilde{Z}$ can only jump at most once on the interval $[k h,(k+1) h]$ for all $k<t L^{6}$. Now we choose

$\Pi_{h}\left(\frac{z}{L}, \frac{z+e_{i}}{L}\right)=L^{-2} R\left(\frac{x+e_{i} / 2}{L}\right), \quad \Pi_{h}\left(\frac{z}{L}, \frac{z}{L}\right)=1-L^{-2} \sum_{e_{i}} R\left(\frac{x+e_{i} / 2}{L}\right)$

for any $z \in \mathbb{Z}^{d}$ and any unit vector $e_{i}$. With this choice, we easily see that $a^{i i}(x)=2 R(x)$ and $b^{i}(x)=\frac{\partial}{\partial x_{i}} R(x)$ at the end of page 267 in [SV07]. Applying Theorem 11.2.3, the Lemma follows.

In case of Theorem 2(a), we consider the first coordinate of $\tilde{Z}$ and the other $d-1$ coordinates separately. Under diffusive scaling, the former one converges to a skew Brownian motion by e.g. [ChShY04, while the latter one converges to a $d-1$ dimensional Brownian motion by Donsker's theorem. A slight technical detail is that we need to switch to discrete time so as to apply the result of [ChShY04]. Let us thus define $\boldsymbol{Z}_{1}^{\prime}(k)=\tilde{\boldsymbol{Z}}_{1}\left(\tau_{1, k}\right)$ for non-negative 
integers $k$, where $\tau_{1,0}=0$ and $\tau_{1, k}=\min \left\{t>\tau_{1, k-1}: \tilde{\boldsymbol{Z}}_{1}(t-) \neq \tilde{\boldsymbol{Z}}_{1}(t+)\right\}$. Then by ChShY04 we have that

$$
\left(\frac{\boldsymbol{Z}_{1}^{\prime}\left(s L^{2}\right)}{L}\right)_{0 \leq s \leq 4 r t} \Rightarrow\left(\mathcal{S} B M_{\omega_{1} /\left(\omega_{-1}+\omega_{1}\right)}(s)\right)_{0 \leq s \leq 4 r t},
$$

where $S B M_{\omega_{1} /\left(\omega_{-1}+\omega_{1}\right)}$ is the skew Brownian motion with parameter $\omega_{1} /\left(\omega_{-1}+\right.$ $\left.\omega_{1}\right)$, which is by definition equal to $\mathcal{Z}_{1}(s) / \sqrt{2 r}$. Now observe that by the law of large numbers, the functions $s \mapsto 2 r \tau_{1, s L^{2}} /\left(s L^{2}\right)$ converge in probability to the identity function on $\mathcal{D}[0, t]$. Hence the statement of the Lemma, restricted to the first component, follows. Finally, the other components are independent from the first one and under diffusive scaling, they converge to Brownian motion by Donsker's theorem.

In case of Theorem 2(b) we use a similar argument to the one in the previous paragraph. Namely, we still have the analogue of (11) and by independence

$$
\left(\frac{\boldsymbol{Z}^{\prime}\left(s L^{2}\right)}{L}\right)_{0 \leq s \leq T} \Rightarrow\left(\mathcal{S} B M_{r_{1} /\left(r_{-1}+r_{1}\right)}(s / d), \mathcal{W}_{d-1}(s / d)\right)_{0 \leq s \leq T},
$$

where

$$
T=4 d \max \left\{r_{-1}, r_{1}\right\} t,
$$

$\mathcal{W}_{d-1}$ is a $d-1$ dimensional standard Brownian motion and $\boldsymbol{Z}^{\prime}(k)=\tilde{\boldsymbol{Z}}\left(\tau_{k}\right)$ with $\tau_{0}=0$ and $\tau_{k}=\min \left\{t>\tau_{k-1}: \tilde{\boldsymbol{Z}}(t-) \neq \tilde{\boldsymbol{Z}}(t+)\right\}$ for positive integers $k$. The difference from the case of Theorem 2 (a) is that now in order to recover the convergence of $\tilde{\boldsymbol{Z}}$, we need a nonlinear time change (and consequently have to work with all coordinates at the same time). To do so, let us introduce the local time the first coordinate spends on the negative and positive halfline:

$$
\tau_{-}(s)=\int_{0}^{s} 1_{f_{1}(y)<0} d y, \quad \tau_{+}(s)=\int_{0}^{s} 1_{f_{1}(y)>0} d y,
$$

for $f=\left(f_{1}, \ldots, f_{d}\right) \in \mathcal{D}\left([0, T], \mathbb{R}^{d}\right)$. Now we can define

$$
\rho(s)=\min \left\{y: \frac{\tau_{-}(y)}{2 d r_{-}}+\frac{\tau_{+}(y)}{2 d r_{+}} \geq s\right\}
$$

and

$$
\Theta: \mathcal{D}([0, T]) \rightarrow \mathcal{D}([0,3 t / 2])
$$

by

$$
(\Theta(f))(s)= \begin{cases}f(\rho(s)) & \text { if } \tau_{-}(T)+\tau_{+}(T) \geq 3 T / 4 \\ 0 & \text { otherwise. }\end{cases}
$$

The function $\Theta$ is well defined because of the choice of $T$. Let us denote by $Q_{L}$ and $Q$ the measure on $\mathcal{D}[0, T]$ given by the left and right hand sides of (12). Next, we observe that

$$
Q\left(\tau_{-}(T)+\tau_{+}(T)=T\right)=1
$$


and $\Theta$ is continuous on the set $\left\{\tau_{-}(T)+\tau_{+}(T)=T\right\}$. Now we can apply the continuous mapping theorem (see e.g. Theorem 5.1 in [B68]) to conclude $\Theta_{*} Q_{L} \Rightarrow \Theta_{*} Q$. Here, $\Theta_{*} Q_{L}$ is the distribution of a process $(\hat{\boldsymbol{Z}}(s))_{0 \leq s \leq 3 t / 2}$ obtained from $\left(\boldsymbol{Z}^{\prime}(s)\right)_{0 \leq s \leq T}$ by rescaling time with $\Theta$. Since $\Theta_{*} Q$ is the distribution of $\mathcal{Z}(s)$, it suffices to show that

$$
\left(\frac{\tilde{\boldsymbol{Z}}\left(s L^{2}\right)-\hat{\boldsymbol{Z}}\left(s L^{2}\right)}{L}\right)_{0 \leq s \leq t} \Rightarrow 0
$$

Let us introduce the notations $z_{k}=\boldsymbol{Z}^{\prime}(k)-\boldsymbol{Z}^{\prime}(k-1), \mathcal{T}_{i}=\tau_{i}-\tau_{i-1}$. By an elementary estimate on the local time of random walks, we have

$$
\mathbb{P}\left(\#\left\{k<t L^{2}: Z^{\prime}(k)_{1}=0\right\}>L^{3 / 2}\right)=o(1) .
$$

Since we prove weak convergence, we can neglect the above event and subsequently assume that $z_{1}, \ldots, z_{t L^{2}}$ is such that the first coordinate's local time at zero is smaller than $L^{3 / 4}$. Especially, we use the first line of the definition of $\Theta$ when we construct $\hat{\boldsymbol{Z}}$ for $L$ large. Thus with the notation

$$
\tilde{i}_{s}=\max \left\{i: \sum_{j=1}^{i} \mathcal{T}_{j}<s\right\} \quad \hat{i}_{s}=\max \left\{i: \sum_{j=1}^{i} \mathbb{E}\left(\mathcal{T}_{j} 1_{\left\{\boldsymbol{Z}^{\prime}(j)_{1} \neq 0\right\}} \mid z_{1}, \ldots, z_{t L^{2}}\right)<s\right\} .
$$

we have $\tilde{\boldsymbol{Z}}(s)=\boldsymbol{Z}^{\prime}\left(\tilde{i}_{s}\right)$ and $\hat{\boldsymbol{Z}}(s)=\boldsymbol{Z}^{\prime}\left(\hat{i}_{s}\right)$. Now with some fixed small $\delta$ let us write

$$
\sum^{k}=\sum_{i \in\left[k \delta L^{2},(k+1) \delta L^{2}\right]} \text { and } \sum^{k *}=\sum_{i \in\left[k \delta L^{2},(k+1) \delta L^{2}\right], \boldsymbol{Z}(i)_{1} \neq 0}
$$

for $k=1,2, \ldots, T / \delta$. Then by Chebyshev's inequality,

$$
\begin{aligned}
& \mathbb{P}\left(\left|\sum^{k *} \mathcal{T}_{i}-\sum^{k *} \mathbb{E}\left(\mathcal{T}_{i} \mid z_{1}, \ldots, z_{t L^{2}}\right)\right|>\delta^{3} L^{2} \mid z_{1}, \ldots, z_{z_{t L^{2}}}\right) \\
< & \frac{\delta L^{2}}{4 d^{2} \min \left\{r_{1}^{2}, r_{-1}^{2}\right\}} \frac{1}{\delta^{6} L^{4}}<\delta^{10}
\end{aligned}
$$

for $L$ large enough. Furthermore, by our assumption on $z_{1}, \ldots, z_{t L^{2}}$,

$$
P\left(\left|\sum^{k *} \mathcal{T}_{i}-\sum^{k} \mathcal{T}_{i}\right|>\delta^{3} L^{2} \mid z_{1}, \ldots, z_{t L^{2}}\right)<\delta^{10}
$$

also holds for $L$ large. Consequently the random times $\tilde{s}_{l}=\sum_{k=1}^{l} \sum^{k *} \mathbb{E}\left(\mathcal{T}_{i} \mid z_{1}, \ldots, z_{t L^{2}}\right)$ and $\hat{s}_{l}=\sum_{i=1}^{l L^{2} / \delta} \mathcal{T}_{i}$ satisfy

- $0=\tilde{s}_{0}<\tilde{s}_{1}<\tilde{s}_{2} \ldots<\tilde{s}_{T}, \tilde{s}_{T} \geq 3 t / 2$ and $\tilde{s}_{l}-\tilde{s}_{l-1}<C \delta$

- for all $l$ with $\tilde{s}_{l}<t, \mathbb{P}\left(\left|\hat{s}_{l}-\tilde{s}_{l}\right|>2 \delta^{2}\right)<\delta^{8}$ and $\hat{\boldsymbol{Z}}\left(\hat{s}_{l} L^{2}\right)=\tilde{\boldsymbol{Z}}\left(\tilde{s}_{l} L^{2}\right)$

This together with tightness of $\boldsymbol{Z}^{\prime}$ (proved in the usual way) gives (13). We have finished the proof of Lemma 2 , 
Lemma 3. If $N=1$, then

$$
\left(\frac{\tilde{\boldsymbol{Y}}^{(L)}\left(s L^{2}\right)}{L}\right)_{0 \leq s \leq t} \Rightarrow(\mathcal{Y}(s))_{0 \leq s \leq t}
$$

Proof. This is a consequence of the continuous mapping theorem, Lemma 2 , (9) and (10).

Next, we prove a simpler version of Theorems 1 and 2, namely the convergence of the expectations

Proposition 5. Consider the setup of either Theorem 1 or Theorem 2. Then

$$
\lim _{L \rightarrow \infty} \mathbb{E}\left(\xi_{\langle x L\rangle}^{(L)}\left(t L^{2}\right)\right)=u(t, x) \frac{\omega_{x}}{2},
$$

where $\omega_{x}=\omega$ in case of Theorems 1 and 2(b) and $=\omega_{\left.\text {sign(x } x_{1}\right)}$ in case of Theorem $2(a)$

Proof. By Proposition 1, the left hand side of (14) is equal to

$$
\frac{\omega_{\langle x L\rangle}}{2} \mathbb{E}\left(\xi_{\tilde{\boldsymbol{Y}}\left(t L^{2}\right)}^{*} \frac{2}{\omega_{\tilde{\boldsymbol{Y}}\left(t L^{2}\right)}} 1_{\left\{\tilde{\boldsymbol{Y}}\left(t L^{2}\right) \in \mathcal{D}_{L}\right\}}+T\left(\frac{\tilde{\boldsymbol{Y}}\left(t L^{2}\right)}{L}\right) 1_{\left\{\tilde{\boldsymbol{Y}}\left(t L^{2}\right) \in \mathcal{B}_{L}\right\}}\right)=: I+I I,
$$

where $\xi_{v}^{*}=\xi_{v}(0)$. In case of Theorems 1 and 2(b), $\omega$ is constant, thus we obtain

$$
I=\sum_{v \in \mathcal{D}_{L}} \mathbb{P}\left(\tilde{\boldsymbol{Y}}\left(t L^{2}\right)=v\right) \mathbb{E}\left(\xi_{v}^{*}\right)
$$

Recall that (7) gives $\mathbb{E}\left(\xi_{v}^{*}\right)=\frac{\omega}{2} f(v / L)+o(1)$. Applying Lemma 3 and the definition of the weak convergence, we obtain

$$
\lim _{L} I=\frac{\omega}{2} \mathbb{E}\left(f(\mathcal{Y}(t)) 1_{\{\mathcal{Y}(t) \in \mathcal{D}\}}\right)
$$

Similarly,

$$
\lim _{L} I I=\frac{\omega}{2} \mathbb{E}\left(T(\mathcal{Y}(t)) 1_{\{\mathcal{Y}(t) \in \partial D\}}\right)
$$

Now the proposition follows from the fact that $u(t, x)$ solves the Kolmogorov equation associated with the process $\mathcal{Y}$. Finally, in case of Theorem 2(a), we consider the function $h$ on $\mathcal{D}$ with $h(y)=0$ if $y_{1}<0, h(y)=y_{1} / \delta$ if $0 \leq y_{1}<\delta$ and $h(y)=1$ if $y_{1}>\delta$ and approximate $I$ by $I a+I b$, where $I a$ is obtained from $I$ by multiplying the integrand with $h\left(\tilde{\boldsymbol{Y}}\left(t L^{2}\right) / L\right)$ and $I b$ is obtained from $I$ by multiplying the integrand with $h\left(-\tilde{\boldsymbol{Y}}\left(t L^{2}\right) / L\right)$. Clearly, $I-\varepsilon<I a+I b<I$ for $\delta$ small enough. Then we can repeat the above argument since $\omega$ is constant on the integration domain in both $I a$ and $I b$.

In order to complete the proof of Theorems 1 and 2 , we need an extension of Lemma 3 from $N=1$ to arbitrary $N$ : 
Proposition 6. The processes

$$
\left(\frac{\tilde{\boldsymbol{Y}}_{i}^{(L)}\left(s L^{2}\right)}{L}\right)_{0 \leq s \leq t}
$$

for $i=1, \ldots, N$ converge weakly to $N$ independent copies of $(\mathcal{Y}(s))_{0 \leq s \leq t}$.

Now, we prove Theorems 1 and 2 assuming Proposition 6. By the discussion at the beginning of this section and by (11), Theorems 11 and 2 will be proved once we establish

$$
\mathbb{E}\left(\prod_{s \in S} \xi_{\langle x L\rangle+s}^{n_{s}^{*}}\left(t L^{2}\right)\right) \sim u(t, x)^{N} \prod_{s \in S} \frac{\Gamma\left(n_{s}^{*}+\omega_{\langle x L\rangle+s} / 2\right)}{\Gamma\left(\omega_{\langle x L\rangle+s} / 2\right)} .
$$

By Proposition 6, $\lim _{L} \mathbb{P}\left(\mathcal{A}_{\delta}^{(L)}\right)=o_{\delta}(1)$, where

$$
\mathcal{A}_{\delta}=\mathcal{A}_{\delta}^{(L)}=\left\{\left\|\tilde{\boldsymbol{Y}}_{i}^{(L)}\left(t L^{2}\right)-\tilde{\boldsymbol{Y}}_{j}^{(L)}\left(t L^{2}\right)\right\| \leq \delta L \text { for some } i \neq j\right\}
$$

This, combined with the uniform moment condition and the Cauchy-Schwarz inequality gives

$$
\lim _{L} \mathbb{E}\left(1_{\mathcal{A}_{\delta}} F\left(\boldsymbol{Y}\left(t L^{2}\right), \underline{\xi}^{*}\right)\right)=o_{\delta}(1),
$$

where $\xi_{v}^{*}=\xi_{v}(0)$ and $F$ is the duality function defined in (41). Now Proposition 1 and the fact that $\underline{\xi}^{*}$ is associated with $f$ implies that the left hand side of (15) is $o_{\delta}(1)$ close to

$$
\begin{aligned}
& \prod_{s \in S} \frac{\Gamma\left(n_{s}^{*}+\omega_{\langle x L\rangle+s} / 2\right)}{\Gamma\left(\omega_{\langle x L\rangle+s} / 2\right)} \times \\
\times & \mathbb{E}\left[1_{\overline{\mathcal{A}}_{\delta}} \prod_{i=1}^{N}\left(\xi_{\tilde{\boldsymbol{Y}}\left(t L^{2}\right)}^{*} \frac{2}{\omega_{\tilde{\boldsymbol{Y}}\left(t L^{2}\right)}} 1_{\left\{\tilde{\boldsymbol{Y}}\left(t L^{2}\right) \in \mathcal{D}_{L}\right\}}+T\left(\frac{\tilde{\boldsymbol{Y}}\left(t L^{2}\right)}{L}\right) 1_{\left\{\tilde{\boldsymbol{Y}}\left(t L^{2}\right) \in \mathcal{B}_{L}\right\}}\right)\right] .
\end{aligned}
$$

Now we can cut this integral to $2^{N}$ pieces and apply a version of the proof of Proposition 5 to conclude (15).

In order to complete the proof of Theorems 1 and 2 it only remains to prove Proposition 6, which is the subject of the next section.

\section{Proof of Proposition 6}

We are going to prove a variant of Proposition [6 obtained by replacing $\tilde{\boldsymbol{Y}}_{i}$ and $\mathcal{Y}$ with $\tilde{Z}$ and $\mathcal{Z}$. Proposition 6 follows from this variant the same way as Lemma 3 follows from Lemma 2.

The idea of the proof is borrowed from [LNY15], Section 5: we show that with probability close to $1, \tilde{\boldsymbol{Z}}_{i}(s)$ and $\tilde{\boldsymbol{Z}}_{j}(s)$ will not meet after getting separated by a distance $L^{\gamma}$ with some $\gamma$ close to 1 . Since $\tilde{\boldsymbol{Z}}_{i}(s)$ and $\tilde{\boldsymbol{Z}}_{j}(s)$ move independently if their distance is bigger than 1 , we can replace $\tilde{\boldsymbol{Z}}(s)$ for $s>\tau=\max \left\{\tau_{i, j}\right\}$ by $N$ independent copies of $\tilde{\boldsymbol{Z}}_{1}(s)$, where $\tau_{i, j}$ is the first time $s$ when $\left\|\tilde{\boldsymbol{Z}}_{i}(s)-\tilde{\boldsymbol{Z}}_{j}(s)\right\|>L^{\gamma}$. It only remains to show that $\tilde{\boldsymbol{Z}}_{i}(\tau)$ is 
close to $x L$ and $\tau / L^{2}$ is negligible. We complete this strategy in the case of Theorem 1, $N=2$ and $d=2$ in Section 6.1 and for all other cases in Section 6.2 .

6.1. Case of Theorem 1, $N=2$ and $d=2$. Recalling the notation of Section [5, let us write $Z(k)=\boldsymbol{Z}_{2}^{\prime}(k)-\boldsymbol{Z}_{1}^{\prime}(k)$. Note that $Z$ is not a particularly nice process: it is neither Markov, nor translation invariant. As long as both $\boldsymbol{Z}_{1}^{\prime}(k)$ and $\boldsymbol{Z}_{2}^{\prime}(s)$ are $\varepsilon L$-close to $x L, Z$ is well approximated by a Brownian motion. In particular, if $\|Z\|=M$, then the probability that $\|Z\|$ reaches $M / 2$ before reaching $2 M$ is close to $1 / 2$ and thus $\|Z\|$ performs an approximate simple symmetric random walk (SSRW) on the circles

$$
\mathcal{C}_{m}=\left\{z \in \mathbb{Z}^{2}:\left|\|z\|-2^{m}\right|<2\right\} .
$$

We will estimate the goodness of this approximation by a SSRW.

Assuming that $Z\left(s_{0}\right) \in \mathcal{C}_{m}$, denote by $s_{1}$ the smallest $s>s_{0}$ such that $Z(s) \in \mathcal{C}_{m-1}$ or $Z(s) \in \mathcal{C}_{m+1}$. We also write

$$
\log =\log _{2}, \quad M=2^{m}, \quad \mathcal{E}_{L, M}=\left|\mathbb{P}\left(Z\left(s_{1}\right) \in \mathcal{C}_{m-1}\right)-\frac{1}{2}\right| .
$$

Note that $s_{1}$ is a stopping time with respect to the filtration generated by $\boldsymbol{Z}_{2}^{\prime}(s), \boldsymbol{Z}_{1}^{\prime}(s)$.

We will need a series of lemmas.

Lemma 4. If $\|Z(k)\| \geq 2$, then

$$
\mathbb{P}(Z(k+1)-Z(k)=e)=\frac{1}{4}+O\left(\frac{\|Z(k)\|}{L^{2}}\right)
$$

for $e=(1,0),(-1,0),(0,1),(0,-1)$. Here, the constants involved in $O$ only depend on the $\mathcal{C}^{2}$ norm of $R$.

Proof. Using that $R$ is a $\mathcal{C}^{2}$ function on a compact domain containing $\mathcal{D}$, the lemma follows from the definition.

Lemma 4 enables us to couple $Z(k)$ with a planar SSRW $W(k)$ such that

$$
\mathbb{P}(Z(k)-Z(k-1)=W(k)-W(k-1) \mid Z(k)) \geq 1-\frac{C\|Z(k)\|}{L^{2}} .
$$

We are going to apply such a coupling several times in the forthcoming lemmas.

Lemma 5. There are constants $\varepsilon_{0}>0, C_{0}<\infty$ and $\theta<1$ such that

$$
\mathbb{P}\left(s_{1}>n\right)<C_{0} \theta^{n / M^{2}}
$$

assuming $\left\|Z\left(s_{0}\right)\right\|=M<\varepsilon_{0} L$.

Proof. To verify Lemma 5, we couple $Z(k), k \in\left[s_{0}, s_{0}+M^{2}\right]$ to a SSRW $W(k), k \in\left[s_{0}, s_{0}+M^{2}\right], W\left(s_{0}\right)=Z\left(s_{0}\right)$. By Lemma 4, we can guarantee that $\|Z(k)-W(k)\|<C M^{3} / L^{2}$ for all $k<M^{2}$ with some probability bounded away from zero. Here, $C$ only depends on the $\mathcal{C}^{2}$ norm of $R$. Thus by choosing 
$\varepsilon_{0}$ small, we can guarantee $C M^{3} / L^{2}<M / 10$. Since $W$ is close to a Brownian motion, it reaches $\mathcal{C}_{m-2}$ or $\mathcal{C}_{m+2}$ before $M^{2}$ with some positive probability. Consequently, there is some $p$ independent of $L$ such that $\mathbb{P}\left(s_{1}<M^{2}\right)>p$. Applying this argument in an inductive fashion gives Lemma 5 .

Let us introduce the notation

$$
\underline{\tau}_{\gamma}=\underline{\tau}_{\gamma}(L)=\min \left\{k:\|Z(k)\|>L^{\gamma}\right\}
$$

Now we claim the following

Lemma 6. For every $\gamma<1$ with $1-\gamma$ small, there exists some $\xi>0$ such that for L large enough, the following estimates hold.

(a) Tiny gap: If $m<\frac{3}{5} \log L$, then

$$
\mathbb{P}\left(\tau_{3 / 5}>L^{13 / 10}\right)=O\left(L^{-1 / 10}\right)
$$

(b) Small gap:

$$
\text { If } \frac{3}{10} \log L \leq m<\gamma \log L, \text { then } \mathcal{E}_{L, M}=O\left(L^{-\xi}\right)
$$

(c) Moderate gap:

$$
\text { If } \gamma \log L<m<\log L-\log \log L \text {, then } \mathcal{E}_{L, M}=O\left(\log ^{-3 / 2} L\right)
$$

(d) Large gap: There is some $\varepsilon>0$ such that

$$
\text { if } \log L-\log \log L<m<\log L+\log \varepsilon \text {, then } \mathcal{E}_{L, M}<1 / 100
$$

As the proof of Lemma 6 is slightly longer than the other lemmas, we postpone it to the Appendix. Next, we formulate our key lemma:

Lemma 7. For any small $\delta>0$, there exists $\gamma<1$ such that for $L$ large enough,

$$
\mathbb{P}\left(\nexists k: \underline{\tau}_{\gamma}<k<t L^{2}:\|Z(k)\| \leq 2\right)>1-\delta .
$$

Proof. In order to derive Lemma 7 from Lemma 6, recall the connection of random walks and electrical networks from Section 1.1. Using the notation from there, we choose $A=\frac{3}{10} \log L, B=\log L+\log \varepsilon$ and $I=\gamma \log L, R_{I+1 / 2}=$ 1. If $R_{i+1 / 2}$ is defined for some $i \in[I, \log L-\log \log L]$, then let us define $R_{i+3 / 2}=R_{i+1 / 2}\left(1+K \log ^{-3 / 2} L\right)$. If $R_{i+1 / 2}$ is defined for some $i \in[\log L-$ $\log \log L, B-1]$, then let us define $R_{i+3 / 2}=\frac{11}{10} w_{i+1 / 2}$. Similarly, if $R_{i+1 / 2}$ is defined for some $i \in[A, I]$, then we define $R_{i-1 / 2}=R_{i+1 / 2}\left(1-K L^{-\xi}\right)$. Now by Lemma 6 (b-d)

$\mathbb{P}\left(\min \left\{k:\left\|Z\left(k+s_{0}\right)\right\|<L^{3 / 10}\right\}<\min \left\{k:\left\|Z\left(k+s_{0}\right)\right\|>\varepsilon L\right\} \mid\left\|Z\left(s_{0}\right)\right\|=L^{\gamma}\right)$

is bounded from above by (2). An elementary computation shows that (2) can be made arbitrarily small by choosing $\gamma$ close to 1 . Thus after $\underline{\tau}_{\gamma},\|Z(k)\|$ reaches $\varepsilon L$ before reaching 2 with probability close to 1 . Finally, Lemmas 1 and 2 yield that for fixed $\varepsilon$ the two particles do not meet after separating by a distance $\varepsilon L$ and before $t L^{2}$ with probability close to 1 . This proves Lemma 7. 
As a consequence of Lemma 7, we will be able to replace $\tilde{\boldsymbol{Z}}_{1}(s)$ and $\tilde{\boldsymbol{Z}}_{2}(s)$ with two independent copies after time

$$
\underline{\tilde{\tau}}_{\gamma}=\min \left\{s:\left\|\tilde{\boldsymbol{Z}}_{1}(s)-\tilde{\boldsymbol{Z}}_{2}(s)\right\|>L^{\gamma}\right\} .
$$

Then Proposition [6] will easily follow once we establish that (A) $\tilde{\tau}_{\gamma} / L^{2}$ is negligible and (B) $\left(\tilde{\boldsymbol{Z}}_{i}\left(\underline{\tau}_{\gamma}\right)-x L\right) / L$ is negligible. This is what we do in the next two lemmas.

Lemma 8. For any fixed $\gamma<1$ and $\delta>0$, we have

$$
\mathbb{P}\left(\underline{\tau}_{\gamma}<L^{1+\gamma}\right)>1-\delta
$$

for L large enough.

Proof. By Lemma 6 (a), it is enough to prove $\mathbb{P}\left(\underline{\tau}_{\alpha}-\underline{\tau}_{3 / 5}>L^{1+\gamma}\right)<\delta$. We write $s_{0}=\underline{\tau}_{3 / 5}$ and if $Z\left(s_{i}\right) \in \mathcal{C}_{m}$ with $m>\frac{3}{10} \log L$, then $s_{i+1}$ is the first time $s$ when either $Z(s) \in \mathcal{C}_{m-1}$ or $Z(s) \in \mathcal{C}_{m+1}$. If $Z\left(s_{i}\right) \in \mathcal{C}_{\left\lfloor\frac{3}{10} \log L\right\rfloor}$, then $s_{i+1}$ is the first time $s$ when $Z(s) \in \mathcal{C}_{\left\lfloor\frac{3}{10} \log L\right\rfloor+1}$. By Lemma 6(b), $b_{i}:=\log \left\|Z\left(s_{i}\right)\right\|$ can be approximated by a one dimensional SSRW (reflected at $\frac{3}{10} \log L$, absorbed at $\gamma \log L)$ with an error of $O\left(L^{\xi}\right)$ at each step. In particular, if $\boldsymbol{t}$ is the smallest $i$ when $b_{i} \geq \gamma \log L$, then $\mathbb{P}\left(\boldsymbol{t}>\log ^{3} L\right)<\delta / 10$ and thus $b_{i}, i \leq \boldsymbol{t}$ can be coupled to a SSRW with an error $<\delta / 5$. Now if $\zeta=(1-\gamma) / 2$ and $\ell_{m}=\#\left\{i<\boldsymbol{t}: b_{i}=m\right\}$, then

$$
\mathbb{P}\left(\exists m: \ell_{m}>L^{\zeta}\right) \leq \log L \max _{m} \mathbb{P}\left(\ell_{m}>L^{\zeta}\right)<\delta / 10
$$

by the gambler's ruin estimate $\mathbb{P}\left(\ell_{m}>n+1 \mid \ell_{m}>n\right)<1-\log ^{-1} L$. If $\ell_{m}<L^{\zeta}$ for all $m$, then

$$
\underline{\tau}_{\alpha}<\sum_{m=\frac{3}{10} \log L}^{\gamma \log L} \sum_{i=1}^{L^{\zeta}} \mathcal{T}_{m, i},
$$

where $\mathcal{T}_{m, 1}$ is the random time $s_{1}-s_{0}$ if $\left\|Z\left(s_{0}\right)\right\|=m$ and for fixed $m, \mathcal{T}_{m, i}$ 's are iid. Consequently, we have

$$
\mathbb{P}\left(\underline{\tau}_{\alpha}>L^{1+\gamma}\right)<\frac{3 \delta}{10}+(\log L) L^{\zeta} \max _{m} \mathbb{P}\left(\mathcal{T}_{m, 1}>L^{1+\gamma-\zeta} \log ^{-1} L\right)<\delta
$$

by Lemma 5 and Lemma 6)(a).

Now, with the notation

$$
\tilde{\bar{\tau}}_{\gamma}=\tilde{\bar{\tau}}_{\gamma}(L)=\min \left\{k:\left\|\tilde{\boldsymbol{Z}}_{1}(k)-x L\right\|>L^{\gamma} \text { or }\left\|\tilde{\boldsymbol{Z}}_{2}(k)-x L\right\|>L^{\gamma}\right\}
$$

we have

Lemma 9. For any fixed $\gamma<1$ and $\delta>0$, we have

$$
\mathbb{P}\left(L^{1+\gamma}<\tilde{\bar{\tau}}_{\frac{\gamma+3}{4}}\right)>1-\delta
$$

for L large enough. 
Proof. By Lemma 1, it is enough to consider the case of one particle. A simplified version of Lemma 2 yields that

$$
\max _{s<L^{1+\gamma}}\left\|\tilde{Z}_{1}(s)-x L\right\|<K L^{\frac{1+\gamma}{2}}
$$

with probability $1-\delta$ for some $K$ and $L$ large.

The variant of Proposition 6 (explained in the beginning of Section 6) easily follows from Lemmas 7, 8 and 9, Since we can neglect an event of small probability, we can assume that all events hold in Lemmas 7, 8 and 9, Note that by definition, $\tilde{\boldsymbol{Z}}_{1}(s)$ and $\tilde{\boldsymbol{Z}}_{2}(s)$ move independently if their distance is bigger than 2. Thus for $s>\tilde{\tilde{\tau}}_{\gamma}$, we can replace $\left(\tilde{\boldsymbol{Z}}_{1}\left(\underline{\tilde{\tau}}_{\gamma}+k\right)\right)_{k=1, \ldots, T L^{2}}$ and $\left(\tilde{\boldsymbol{Z}}_{2}\left(\underline{\tilde{\tau}}_{\gamma}+k\right)\right)_{k=1, \ldots, T L^{2}}$ with independent random walks, both of them converging to $\mathcal{Z}(s)$ under the proper scaling. Finally, by Lemmas 8 and 9 , $\underline{\tau}_{\gamma} / L^{2}<\delta$ and $\left(\tilde{\boldsymbol{Z}}_{i}\left(\underline{\tilde{\tau}}_{\gamma}\right)-x\right) / L<\delta$. Proposition 6 follows in the case of Theorem 1, $N=2$, $d=2$.

6.2. Completing the proof of Proposition 6. The case of general $N$ follows from Lemma 1 and from the case $N=2$. Indeed, Lemmas 1, 7 and 8 imply that for some $\gamma<1$,

$$
\mathbb{P}\left(\exists s \in\left[L^{1+\gamma}, t L^{2}\right], i, j \in\{1,2, \ldots, N\}:\left\|\tilde{\boldsymbol{Z}}_{i}(s)-\tilde{\boldsymbol{Z}}_{j}(s)\right\| \leq 2\right)<\delta .
$$

Furthermore, we have the analogue of Lemma 9 with $\tilde{\bar{\tau}}_{\alpha}$ replaced by

$$
\min \left\{k: \exists i<N:\left\|\tilde{\boldsymbol{Z}}_{i}(k)-x L\right\|>L^{\alpha}\right\} .
$$

Whence the case of general $N$ follows the same way as before.

The case of dimension $d>2$ is simpler than $d=2$ as the particles only meet finitely many times.

Lemma 10. In case of Theorem 1, $d>2, N=2$, there is some positive $p$ such that $\left\|\tilde{\boldsymbol{Z}}_{1}(s)-\tilde{\boldsymbol{Z}}_{2}(s)\right\| \geq 2$ for all $k \in\left[2, t L^{2}\right]$ with probability at least $p$.

Proof. Consider the process $Z(k)=\boldsymbol{Z}_{2}^{\prime}(k)-\boldsymbol{Z}_{1}^{\prime}(k)$ as in Section 6.1. Let us fix some small $\varepsilon>0$. We will show that the probability of the event $\{\|Z\|$ reaches $\varepsilon L$ before reaching 1$\}$ is bounded away from zero. From this the lemma will follow by the same argument as in $d=2$ (cf. the end of the proof of Lemma 7).

Similarly to Lemma 4, we have

$$
\frac{1}{2 d}-\frac{c_{0} \varepsilon}{L}<\mathbb{P}(Z(k+1)-Z(k)=e)<\frac{1}{2 d}+\frac{c_{0} \varepsilon}{L}
$$

assuming $\|Z(k)\| \leq \varepsilon L$ for all unit vectors $e \in \mathbb{Z}^{d}$ and $L$ large enough. Now we define a random walk $B(k)$ on $\mathbb{Z}^{d}$ with weights. Specifically $B(3)=Z(3)$ and we choose the weights $w_{(u, v)}=\left(1-\frac{c_{1} \varepsilon}{L}\right)^{l}$, where $|u|_{1}=l-1,|v|_{1}=l$ and $c_{1} \gg c_{0}$ is a fixed constant. 
Clearly $\|Z(3)\| \geq 2$ holds with some positive probability. Let us write $\boldsymbol{t}_{B, l}=$ $\min \left\{k>3:|B(k)|_{1}=l\right\}$, where $\min \emptyset=\infty$ and similarly $\boldsymbol{t}_{Z, l}=\min \{k>3$ : $\|Z(k)\|=l\}$. Now we claim that

Lemma 11. Assuming $c_{1}=c_{1}\left(c_{0}\right)$ is large enough, there exists a coupling between the processes $B$ and $Z$ such that $\left|B_{i}(k)\right| \leq\left|Z_{i}(k)\right|$ holds for all $k \in$ $\left[3, \boldsymbol{t}_{B, 1} \wedge \boldsymbol{t}_{Z, \varepsilon L}\right)$ and $i \leq d$ almost surely.

By Lemma 11, it suffices to prove that

$$
\mathbb{P}\left(\boldsymbol{t}_{B, \varepsilon L}<\boldsymbol{t}_{B, 1}\right) \text { is bounded away from zero. }
$$

This follows from a simple application of the connection between random walks and electrical networks. Note that the weights $w_{(u, v)}$ are bounded away from zero in the $\varepsilon L$ neighborhood of the origin. In such cases, (18) follows from a standard argument, see e.g. the proof of Theorem 19.30 in [K14]. In order to complete the proof of the Lemma 10, it only remains to prove Lemma 11.

Proof of Lemma 11. We prove by induction on $k$. Assume $\left|B_{i}(k)\right| \leq\left|Z_{i}(k)\right|$ for all $i \leq d$.

Case 1: none of the coordinates of $B(k)$ and $Z(k)$ are zero.

If $\left|B_{i}(k+1)\right|=\left|B_{i}(k)\right|+1$, then we define $Z(k+1)$ by $\left|Z_{i}(k+1)\right|=\left|Z_{i}(k)\right|+1$. We can do so by (17) and by the definition of $B$ : if $c_{1}$ is large enough, then

$$
\mathbb{P}\left(\left|B_{i}(k+1)\right|=\left|B_{i}(k)\right|+1\right) \leq \mathbb{P}\left(\left|Z_{i}(k+1)\right|=\left|Z_{i}(k)\right|+1\right) .
$$

Similarly, if $\left|Z_{i}(k+1)\right|=\left|Z_{i}(k)\right|-1$, then we define $B(k+1)$ by $\left|B_{i}(k+1)\right|=$ $\left|B_{i}(k)\right|-1$. We can do so as similarly to (19), we have

$$
\mathbb{P}\left(\left|Z_{i}(k+1)\right|=\left|Z_{i}(k)\right|-1\right) \leq \mathbb{P}\left(\left|B_{i}(k+1)\right|=\left|B_{i}(k)\right|+1\right) .
$$

The coupling is arbitrary on the remaining set (sometimes we may have to move $B$ and $Z$ in different directions to match the probabilities, i.e. to define a proper coupling). This proves the inductive step for the case when none of the coordinates of $B(k)$ and $Z(k)$ are zero.

Case 2: none of the coordinates of $B(k)$ are zero or for all $i$ with $B_{i}(k)=0$, $Z_{i}(k)=0$ also holds.

The same argument works as in Case 1.

Note that the coupling of Case 1 will not work if $B_{i}(k)=0$ and $Z_{i}(k) \neq 0$ as $\mathbb{P}\left(\left|B_{i}(k+1)\right|=\left|B_{i}(k)\right|+1\right) \approx d^{-1}$ while $\mathbb{P}\left(\left|Z_{i}(k+1)\right|=\left|Z_{i}(k)\right|+1\right) \approx(2 d)^{-1}$. Let $\mathcal{I}$ denote the set of indices $i$ with $B_{i}(k)=0, Z_{i}(k) \neq 0$ and let $\mathcal{I}^{\prime} \subset \mathcal{I}$ be the set of indices $i$ with $B_{i}(k)=0$ and $\left|Z_{i}(k)\right|=1$.

Case 3: $\left|Z_{i}(k)\right| \geq 2$ for all $i \in \mathcal{I}$. If $\left|B_{i}(k+1)\right|=\left|B_{i}(k)\right|+1$ with some $i \in \mathcal{I}$, then we can define $Z(k+1)$ by changing the $i$ th coordinate (either increasing or decreasing), since we have

$$
\begin{aligned}
& \mathbb{P}\left(B(k+1)=B(k)+e_{i} \text { or } B(k)-e_{i}\right) \\
\leq & \mathbb{P}\left(Z(k+1)=Z(k)+e_{i} \text { or } Z(k)-e_{i}\right) .
\end{aligned}
$$


The proof of (20) is similar to that of (19): the first line of (20) is

$$
\frac{w_{\left(B(k), B(k)+e_{i}\right)}+w_{\left(B(k), B(k)-e_{i}\right)}}{\sum_{v:\|v-B(k)\|=1} w_{(B(k), v)}} .
$$

Here the denominator can be bounded from below by

$$
\left(1-\frac{c_{1} \varepsilon}{L}\right)^{|B(k)|_{1}}\left[1+(2 d-1)\left(1-\frac{c_{1} \varepsilon}{L}\right)\right],
$$

which corresponds to the case when only one coordinate is nonzero (note that we have excluded $B(k)=0$ since $\left.k<\boldsymbol{t}_{B, 1}\right)$. Combining this estimate with (17) gives (20) assuming that $c_{1}=c_{1}\left(c_{0}\right)$ is large enough. The other coordinates are treated the same way as in Case 1.

Case 4: $\left|\mathcal{I}^{\prime}\right| \neq 0$ is an even integer.

Consider a perfect matching of $\mathcal{I}^{\prime}$. Let $(i, j) \in \mathcal{I}^{\prime 2}$ be an arbitrary pair. If $Z_{i}(k+1)=0$, then we define $B(k+1)$ such that $\left|B_{j}(k+1)\right|=1$. We can do so, since $\mathbb{P}\left(Z_{i}(k+1)=0\right) \approx(2 d)^{-1}$ and $\mathbb{P}\left(\left|B_{j}(k+1)\right|=1\right) \approx d^{-1}$. Analogously, if $Z_{j}(k+1)=0$, then we define $B(k+1)$ such that $\left|B_{i}(k+1)\right|=1$. Then, we do the coupling of movements in other directions as discussed in cases 1-3. Finally, we couple the remaining set (including $Z_{i}(k+1)=2, Z_{j}(k+1)=2$ ) arbitrarily.

Case 5: $\left|\mathcal{I}^{\prime}\right|=n$ is an odd integer.

First we consider a matching of $n-1$ elements of $\mathcal{I}^{\prime}$, and do the coupling described in Case 4. Let us denote the remaining index by $i$. Now we claim that there is some $j \notin \mathcal{I}^{\prime}$ such that $\left|Z_{j}(k)\right|-\left|B_{j}(k)\right| \geq 1$. Indeed, if there was no such $j$, then $\sum_{m=1}^{d}\left|Z_{m}(k)\right|-\left|B_{m}(k)\right|=n$ would be an odd number, which is a contradiction with the fact that $Z(0)=B(0)$ and both processes move to nearest neighbors at each step. Now if $\left|Z_{j}(k+1)\right|=\left|Z_{j}(k)\right|-1$, then we define $B(k+1)$ such that $\left|B_{i}(k+1)\right|=1$. If $Z_{i}(k+1)=0$, we define $B(k+1)$ by changing the $j$ th coordinate (either decreasing or increasing). These can be done as before. Then, we consider the cases corresponding to other coordinates as discussed in cases 1-3. Finally, we couple the remaining set arbitrarily.

Using Lemma 10, one can easily prove a much simplified version of Lemmas 7. 8 and 9 with $L^{\gamma}, L^{\frac{1+\gamma}{2}}, L^{\frac{3+\gamma}{4}}$ replaced by constants $K_{1}(\delta), K_{2}(\delta), K_{3}(\delta)$ This implies Proposition 6 for the case $d>2, N=2$. Then the case of general $N$ follows the same way as in $d=2$.

Finally, in case of Theorem 2 we have assumed that $x_{1} \neq 0$. Then choosing $\varepsilon<\left|x_{1}\right|$, the proof of the case of Theorem 1 (with the choice $R$ is the constant 1 function) applies.

\section{Proof of Theorem 3 and Proposition 4}

As in the proof of Theorems 1 and 2, we prove the weak convergence by showing that the moments converge. The latter one is showed by switching to the dual process. Recalling some notation from Section 5, we define $\boldsymbol{Y}^{\prime}$ from 
$\tilde{\boldsymbol{Y}}(t)$ the same way as we defined $\boldsymbol{Z}^{\prime}$ from $\tilde{\boldsymbol{Z}}(t)$. The proof consists of two parts. First we consider the case when the number of particles is $N=2$ and prove

\section{Proposition 7.}

$$
\lim _{L \rightarrow \infty} \mathbb{E}\left[T\left(\frac{\boldsymbol{Y}_{1}^{\prime(L)}(\infty)}{L}\right) T\left(\frac{\boldsymbol{Y}_{2}^{\prime(L)}(\infty)}{L}\right)\right] /\left[u^{(L)}(x)\right]^{2}=1
$$

Then we can derive an extension of Proposition 7 to arbitrary $N$. The idea of the proof of Proposition 7 is borrowed from [RY07] (the main difference is in Lemma 14). The extension to arbitrary $N$ is very similar to the argument in KMP82, LNY15.

Let us write $P^{(L)}\left(A_{1}\right)=\mathbb{P}\left(\boldsymbol{Y}_{1}^{\prime(L)}(\infty)=A_{1}\right)$ and

$$
P^{(L)}\left(A_{1}, A_{2}\right)=\mathbb{P}\left(\boldsymbol{Y}_{1}^{\prime(L)}(\infty)=A_{1}, \boldsymbol{Y}_{2}^{\prime(L)}(\infty)=A_{2}\right)
$$

for $A_{1}, A_{2} \in\{0, L\}$. The asymptotic hitting probabilities of one particle are given by

Lemma 12. $\lim _{L \rightarrow \infty} \frac{P^{(L)}(L)}{\mathcal{A}^{(L)}(x)}=1$.

Proof. Although this lemma follows from the connection between random walks and electrical networks, we give a direct proof as its extensions will be needed later. Note that by the definition of $\omega_{0}, \omega_{L}$ and $\psi(m)$, we have for any $1 \leq m \leq L-1$

$$
\frac{\omega_{m-1}}{\omega_{m-1}+\omega_{m}} r_{m-1 / 2} \psi(m)=\frac{\omega_{m+1}}{\omega_{m}+\omega_{m+1}} r_{m+1 / 2} \psi(m+1) .
$$

Let us write $\underline{\Phi}(m)=\sum_{i=1}^{m} \psi(i)$ for $0 \leq m \leq L$. Then (21) means that $\underline{\Phi}\left(\boldsymbol{Y}_{1}^{\prime}(k)\right)$ is a bounded martingale. After the first hitting of either 0 or $L$, this martingale clearly stays constant. Then by the martingale convergence theorem,

$$
P^{(L)}(L) \underline{\Phi}(L)=\underline{\Phi}\left(\boldsymbol{Y}_{1}^{\prime}(0)\right) .
$$

Since $\mathcal{A}^{(L)}(x) \sim \underline{\Phi}\left(\boldsymbol{Y}_{1}^{\prime}(0)\right) / \underline{\Phi}(L)$, Lemma 12 follows.

Now with the notation $\bar{\Phi}(m)=\sum_{i=m+1}^{L} \psi(i)$ for $0 \leq m \leq L$ our aim is to construct submartingales using

$$
S_{k}:=\underline{\Phi}\left(\boldsymbol{Y}_{1}^{\prime}(k)\right) \underline{\Phi}\left(\boldsymbol{Y}_{2}^{\prime}(k)\right)+\bar{\Phi}\left(\boldsymbol{Y}_{1}^{\prime}(k)\right) \bar{\Phi}\left(\boldsymbol{Y}_{2}^{\prime}(k)\right)
$$

and

$$
T_{k}=\sum_{i=\min \left\{\boldsymbol{Y}_{1}^{\prime}(k), \boldsymbol{Y}_{2}^{\prime}(k)\right\}+1}^{\max \left\{\boldsymbol{Y}_{1}^{\prime}(k), \boldsymbol{Y}_{2}^{\prime}(k)\right\}} \psi(i)
$$

Lemma 13. There exists some constant $C$ only depending on the upper and lower bound of $r$ and $\omega$ such that $S_{k}+C T_{k}$ is a submartingale and $S_{k}-C T_{k}$ is a supermartingale. 
Proof. Let us compute the conditional expectations with respect to $\left(\mathcal{F}_{k}\right)_{k}$, the filtration generated by the process $\boldsymbol{Y}^{\prime}$. First, observe that $\mathbb{E}\left(S_{k+1} \mid \mathcal{F}_{k}\right)=S_{k}$ if $\left|\boldsymbol{Y}_{1}^{\prime}(k)-\boldsymbol{Y}_{2}^{\prime}(k)\right| \geq 2$. Next, by definition $\mathbb{E}\left(S_{k+1} \mid \boldsymbol{Y}_{1}^{\prime}(k)=\boldsymbol{Y}_{2}^{\prime}(k)=i\right)$ is equal to

$$
\begin{aligned}
\frac{r_{i+1 / 2}}{r_{i-1 / 2}+r_{i+1 / 2}} & \int_{0}^{1} p^{2}\left[\underline{\Phi}(i+1)^{2}+\bar{\Phi}(i+1)^{2}\right] \\
& +2 p(1-p)[\underline{\Phi}(i) \underline{\Phi}(i+1)+\bar{\Phi}(i) \bar{\Phi}(i+1)] \\
& +(1-p)^{2}\left[\underline{\Phi}(i)^{2}+\bar{\Phi}(i)^{2}\right] \operatorname{dBeta}\left(\omega_{i+1} / 2, \omega_{i} / 2, p\right) \\
+\frac{r_{i-1 / 2}}{r_{i-1 / 2}+r_{i+1 / 2}} & \int_{0}^{1} p^{2}\left[\underline{\Phi}(i-1)^{2}+\bar{\Phi}(i-1)^{2}\right] \\
& +2 p(1-p)[\underline{\Phi}(i) \underline{\Phi}(i-1)+\bar{\Phi}(i) \bar{\Phi}(i-1)] \\
& +(1-p)^{2}\left[\underline{\Phi}(i)^{2}+\bar{\Phi}(i)^{2}\right] \operatorname{dBeta}\left(\omega_{i-1} / 2, \omega_{i} / 2, p\right)
\end{aligned}
$$

where we used the shorthand $\operatorname{dBeta}(\alpha, \beta, p)=\frac{1}{B(\alpha, \beta)} p^{\alpha-1}(1-p)^{\beta-1} d p$. Computing the integrals and using (21) gives

$$
\begin{aligned}
& \mathbb{E}\left(S_{k+1}-S_{k} \mid \boldsymbol{Y}_{1}^{\prime}(k)=\boldsymbol{Y}_{2}^{\prime}(k)=i\right) \\
= & 2[\psi(i)]^{2}\left(\frac{r_{i+1 / 2}}{r_{i-1 / 2}+r_{i+1 / 2}} \frac{\omega_{i+1}\left(\omega_{i+1}+2\right)}{\left(\omega_{i}+\omega_{i+1}\right)\left(\omega_{i}+\omega_{i+1}+2\right)}\right. \\
& \left.+\frac{r_{i-1 / 2}}{r_{i-1 / 2}+r_{i+1 / 2}} \frac{\omega_{i-1}\left(\omega_{i-1}+2\right)}{\left(\omega_{i-1}+\omega_{i}\right)\left(\omega_{i-1}+\omega_{i}+2\right)}\right)
\end{aligned}
$$

Similarly, $\mathbb{E}\left(S_{k+1}-S_{k} \mid \boldsymbol{Y}_{1}^{\prime}(k)=i, \boldsymbol{Y}_{2}^{\prime}(k)=i+1\right)$ is by definition equal to

$$
\begin{aligned}
\frac{r_{i-1 / 2}}{r_{i-1 / 2}+r_{i+1 / 2}+r_{i+3 / 2}} & \frac{\omega_{i-1}}{\omega_{i-1}+\omega_{i}}[-\psi(i) \underline{\Phi}(i+1)+\psi(i) \bar{\Phi}(i+1)] \\
+\frac{r_{i+1 / 2}}{r_{i-1 / 2}+r_{i+1 / 2}+r_{i+3 / 2}} & \left\{\int _ { 0 } ^ { 1 } \left(p^{2}\left[\underline{\Phi}(i+1)^{2}+\bar{\Phi}(i+1)^{2}\right]\right.\right. \\
& +2 p(1-p)[\underline{\Phi}(i) \underline{\Phi}(i+1)+\bar{\Phi}(i+1) \bar{\Phi}(i)] \\
& \left.+(1-p)^{2}\left[\underline{\Phi}(i)^{2}+\bar{\Phi}(i)^{2}\right]-S_{k}\right) \operatorname{dBeta}\left(\omega_{i-1}, \omega_{i}, p\right) \\
& \left.-\underline{\Phi}(i)^{2}-\bar{\Phi}(i)^{2}\right\} \\
+\frac{r_{i+3 / 2}}{r_{i-1 / 2}+r_{i+1 / 2}+r_{i+3 / 2}} & \frac{\omega_{i+2}}{\omega_{i+1}+\omega_{i+2}}[\underline{\Phi}(i) \psi(i+2)-\bar{\Phi}(i) \psi(i+2)] .
\end{aligned}
$$

A similar computation to the previous one gives

$$
\begin{aligned}
& \mathbb{E}\left(S_{k+1}-S_{k} \mid \boldsymbol{Y}_{1}^{\prime}(k)=i, \boldsymbol{Y}_{2}^{\prime}(k)=i+1\right) \\
= & -[\psi(i+1)]^{2} \frac{2 r_{i+1 / 2}}{r_{i-1 / 2}+r_{i+1 / 2}+r_{i+3 / 2}} \frac{\omega_{i} \omega_{i+1}}{\left(\omega_{i}+\omega_{i+1}\right)\left(\omega_{i}+\omega_{i+1}+2\right)} .
\end{aligned}
$$

Just like in the case of $S_{k}$, we have $\mathbb{E}\left(T_{k+1} \mid \mathcal{F}_{k}\right)=T_{k}$ if $\left|\boldsymbol{Y}_{1}^{\prime}(k)-\boldsymbol{Y}_{2}^{\prime}(k)\right| \geq 2$. Furthermore,

$$
\mathbb{E}\left(T_{k+1}-T_{k} \mid \boldsymbol{Y}_{1}^{\prime}(k)=\boldsymbol{Y}_{2}^{\prime}(k)=i\right)=[\psi(i+1)] \frac{r_{i+1 / 2}}{r_{i-1 / 2}+r_{i+1 / 2}} \frac{\omega_{i} \omega_{i+1}}{\left(\omega_{i}+\omega_{i+1}\right)},
$$


and

$$
\mathbb{E}\left(T_{k+1}-T_{k} \mid \boldsymbol{Y}_{1}^{\prime}(k)=i, \boldsymbol{Y}_{2}^{\prime}(k)=i+1\right)=[\psi(i+1)] \frac{1}{3} \frac{\omega_{i} \omega_{i+1}}{\left(\omega_{i}+\omega_{i+1}+2\right)} .
$$

We conclude that there is some positive constant $c$ such that

- $0<\mathbb{E}\left(S_{k+1}-S_{k} \mid \boldsymbol{Y}_{1}^{\prime}(k)=\boldsymbol{Y}_{2}^{\prime}(k)\right)<\frac{1}{c}$

- $-1 / c<\mathbb{E}\left(S_{k+1}-S_{k}|| \boldsymbol{Y}_{1}^{\prime}(k)-\boldsymbol{Y}_{2}^{\prime}(k) \mid=1\right)<0$

- $c<\mathbb{E}\left(T_{k+1}-T_{k} \mid \boldsymbol{Y}_{1}^{\prime}(k)=\boldsymbol{Y}_{2}^{\prime}(k)\right)$

- $c<\mathbb{E}\left(T_{k+1}-T_{k}|| \boldsymbol{Y}_{1}^{\prime}(k)-\boldsymbol{Y}_{2}^{\prime}(k) \mid=1\right)$

The lemma follows.

Lemma 14. $\lim _{L \rightarrow \infty} \frac{P^{(L)}(L, L)+P^{(L)}(0,0)}{\left[\mathcal{A}^{(L)}(x)\right]^{2}+\left[1-\mathcal{A}^{(L)}(x)\right]^{2}}=1$.

Proof. Since $M_{k}:=S_{k}+C T_{k}$ is a bounded submartingale, we can apply the martingale convergence theorem to deduce

$M_{0} \leq \mathbb{E}\left(M_{\infty}\right)=\left(P^{(L)}(L, L)+P^{(L)}(0,0)\right) \underline{\Phi}^{2}(L)+\left(P^{(L)}(0, L)+P^{(L)}(L, 0)\right) C \underline{\Phi}(L)$

Since $\Phi(L) / L$ is bounded away from zero and infinity, the lower bound follows. The upper bound is derived similarly from the fact that $S_{k}+C T_{k}$ is a supermartingale.

Now we are ready to prove Proposition 7

Proof of Proposition 7 . By Lemma 1.

$$
P^{(L)}(L, L)=\frac{1}{2}\left[P^{(L)}(L)+P^{(L)}(L, L)+P^{(L)}(0,0)-P^{(L)}(0)\right]
$$

Then by Lemmas 12 and 14 ,

$$
P^{(L)}(L, L) \sim\left[\mathcal{A}^{(L)}(x)\right]^{2} .
$$

Similarly, $P^{(L)}(0,0) \sim\left[1-\mathcal{A}^{(L)}(x)\right]^{2}, P^{(L)}(L, 0) \sim P^{(L)}(0, L) \sim\left[\mathcal{A}^{(L)}(x)\right][1-$ $\mathcal{A}^{(L)}(x)$ ]. Proposition 7 follows.

Since the extension of Proposition 7 to arbitrary $N$ can be proved the same way as its analogues in [KMP82, Section 3 and [LNY15], Section 6.2, we omit the proof here. Thus we have finished the proof of Theorem 3 .

Finally, Proposition 4(b) is elementary and Proposition 4(a) follows from the connection between random walks and electrical networks (namely, from (2) or Lemma 12) and the law of large numbers.

\section{APPENDIX}

Here, we prove Lemma 6. We will use the notations of Section 6. The proof uses similar coupling to the one in the proof of Lemma 5. This will suffice in case of (a), as $\|Z\|$ is small and in case of (d), as we only need a weak estimate. However, we need to perform the coupling on a mesoscopic timescale to complete the proof of (b) and (c). 


\section{Proof of (d).}

We prove the following slightly stronger statement

For every $\eta>0$ there is $\varepsilon=\varepsilon(\eta)>0$ such that if $\log L-\log \log L<m<$ $\log L+\log \varepsilon$ and $L$ is large enough, then $\mathcal{E}_{L, M}<\eta$.

With some fixed $C_{1}$, we couple $Z(k), k \in\left[s_{0},\left(s_{0}+C_{1} M^{2}\right) \wedge s_{1}\right]$ to a SSRW $W(k), k \in\left[s_{0},\left(s_{0}+C_{1} M^{2}\right) \wedge s_{1}\right], W\left(s_{0}\right)=Z\left(s_{0}\right)$. We fix $C_{1}$, as we can by Lemma 5, such that $\mathbb{P}\left(s_{1}>s_{0}+C_{1} M^{2}\right)<\eta / 10$. Second, Lemma 4 implies that by choosing $C_{2}=C_{2}\left(C_{1}\right)$ large enough,

$$
\mathbb{P}\left(\|W(k)-Z(k)\|>C_{2} M^{3} / L^{2} \text { for some } k<\left(s_{0}+C_{1} M^{2}\right) \wedge s_{1}\right)<\eta / 10 .
$$

Now let us define

$$
\begin{aligned}
& \underline{s}_{1}=\min \{k:\|W(k)\|<(1 / 2+\zeta) M \text { or }\|W(k)\|>(2-\zeta) M\}, \\
& \bar{s}_{1}=\min \{k:\|W(k)\|<(1 / 2-\zeta) M \text { or }\|W(k)\|>(2+\zeta) M\}
\end{aligned}
$$

Where $\zeta$ is fixed in such a way that the following events concerning a planar Brownian motion $\mathcal{W}$ have probability at least $1-\eta / 10$ :

(A) if $\|\mathcal{W}(0)\|=1 / 2+\zeta$ then $\mathcal{W}$ reaches $B(0,1 / 2-\zeta)$ before reaching $\mathbb{R}^{2} \backslash B(0,2-\zeta)$ and

(B) if $\|\mathcal{W}(0)\|=2-\zeta$ then $\mathcal{W}$ reaches $\mathbb{R}^{2} \backslash B(0,2+\zeta)$ before reaching $B(0,1 / 2+\zeta)$.

By the fact that $\log \|\mathcal{W}\|$ is martingale and by Donsker's theorem, we can also assume

$$
\left|\mathbb{P}\left(\left\|W\left(\underline{s}_{1}\right)\right\|<(1 / 2+\zeta) M\right)-1 / 2\right|<\eta / 10,
$$

possibly by further reducing $\zeta$ and by choosing $L$ large. Finally, we choose $\varepsilon=\sqrt{\zeta / C_{2}}$ so as $\zeta M>C_{2} M^{3} / L^{2}$. Lemma 6 (d) follows.

\section{Proof of (a)}

If $2 \leq\left\|Z\left(k+s_{0}\right)\right\|$ for some $k<L^{13 / 10} \wedge \underline{\tau}_{3 / 5}$, then using Lemma 4 we can couple $Z(k)-Z(k-1)$ to a step of a SSRW $W(k)-W(k-1)$ with probability $O\left(L^{-7 / 5}\right)$. Thus the probability

$$
\mathbb{P}\left(Z(k)-Z(k-1)=W(k)-W(k-1) \text { for all } k<L^{13 / 10}, 2 \leq\left\|Z\left(k+s_{0}\right)\right\|\right)
$$

is $O\left(L^{-1 / 10}\right)$. Now Lemma 6(a) follows from the proof of Lemmas 10 and 11 in [LNY15].

\section{Proof of (b) and (c)}

The idea of the proof of these cases is borrowed from [DSzV08. First, we fix some positive $\xi<3 / 20$, write $K=\left\lfloor L^{\xi}\right\rfloor$ and consider the process $\left(Z\left(s_{0}+j K\right)\right)_{j \geq 1}$, stopped upon reaching $B(0, M / 2-K)$ or $\mathbb{R}^{2} \backslash B(0,2 M+K)$. Let us denote by $\bar{s}$ the corresponding stopping time, i.e. $\bar{s}=s K$ with the smallest $s$ such that $Z\left(s_{0}+s K\right)$ is stopped. We will also write $\mathfrak{z}=Z\left(s_{0}+\right.$ $j K)-Z\left(s_{0}+(j-1) K\right)$ and $\mathfrak{x}_{j}=\log \left\|Z\left(s_{0}+j K\right)\right\|^{2}$. Now we have

$$
\left\|Z\left(s_{0}+(j+1) K\right)\right\|^{2}=\left\|Z\left(s_{0}+j K\right)\right\|^{2}+2\left(Z\left(s_{0}+j K\right), \mathfrak{z}\right)+\|\mathfrak{z}, \mathfrak{z}\|^{2}
$$


and

$$
\mathfrak{x}_{j+1}-\mathfrak{x}_{j}=\log \left(1+\frac{2\left(Z\left(s_{0}+j K\right), \mathfrak{z}\right)+\|\mathfrak{z}, \mathfrak{z}\|^{2}}{\left\|Z\left(s_{0}+j K\right)\right\|^{2}}\right)
$$

By Taylor expansion,

$$
\begin{aligned}
& \mathfrak{x}_{j+1}-\mathfrak{x}_{j} \\
= & \frac{2\left(Z\left(s_{0}+j K\right), \mathfrak{z}\right)}{\left\|Z\left(s_{0}+j K\right)\right\|^{2}}+\frac{\|\mathfrak{z}\|^{2}}{\left\|Z\left(s_{0}+j K\right)\right\|^{2}}-\frac{2\left(Z\left(s_{0}+j K\right), \mathfrak{z}\right)^{2}}{\left\|Z\left(s_{0}+j K\right)\right\|^{4}}+O\left(L^{3 \xi} M^{-3}\right) \\
= & I+I I+I I I+O\left(L^{3 \xi} M^{-3}\right)
\end{aligned}
$$

As before, we couple $\mathfrak{z}$ to a SSRW $W(K)$ by Lemma 4 such that if $\mathfrak{y}=$ $\mathfrak{z}-W(K)$, then for all positive integer $n$,

$$
\mathbb{P}(\|\mathfrak{y}\|=k)=O\left(L^{n \xi-2 n} M^{n}\right)
$$

If $\mathcal{F}_{k}$ is the filtration generated by $\tilde{\boldsymbol{Z}}_{i}\left(s_{0}+l\right), l \leq k, i=1,2$, then

$$
\mathbb{E}(I)=\mathbb{E}\left(\frac{\mathbb{E}\left(2\left(Z\left(s_{0}+j K\right), W(K)\right) \mid \mathcal{F}_{j}\right)}{\left\|Z\left(s_{0}+j K\right)\right\|^{2}}\right)+\mathbb{E}\left(\frac{\mathbb{E}\left(2\left(Z\left(s_{0}+j K\right), \mathfrak{y}\right) \mid \mathcal{F}_{j}\right)}{\left\|Z\left(s_{0}+j K\right)\right\|^{2}}\right)
$$

Here, the first term is zero by symmetry of the SSRW $W$, and the second is estimated by the Cauchy-Schwarz inequality in cases $1 \leq\|\mathfrak{y}\| \leq 2$ and $\|\mathfrak{y}\| \geq 3$. We conclude $\mathbb{E}(I)=L^{\xi-2}$. A similar argument shows that

$$
\mathbb{E}\left(\|\mathfrak{z}\|^{2}\right)=K+O\left(M L^{3 \xi-2}\right) .
$$

The above leading term is $\mathbb{E}\left(\|W(K)\|^{2}\right)=K$, which can be easily proved by leveraging the fact that $\left(W_{(1)}(k)-W_{(1)}(k-1)\right)_{1 \leq k \leq K}$ and $\left(W_{(2)}(k)-\right.$ $\left.W_{(2)}(k-1)\right)_{1 \leq k \leq K}$ are two independent one dimensional SSRW's, where $v_{(1)}=$ $((1 / \sqrt{2}, 1 / \sqrt{2}), v)$ and $v_{(2)}=((1 / \sqrt{2},-1 / \sqrt{2}), v)$. Finally,

$$
\begin{aligned}
& \mathbb{E}\left(2\left(Z\left(s_{0}+j K\right), \mathfrak{z}\right)^{2} \mid \mathcal{F}_{j}\right) \\
= & \sum_{i, j=1}^{2} 2 Z_{(i)}\left(s_{0}+j K\right) Z_{(j)}\left(s_{0}+j K\right) \mathbb{E}\left(\mathfrak{z}_{(i)} \mathfrak{z}(j)\right) \\
= & \sum_{i=1}^{2} 2\left(Z_{(i)}\left(s_{0}+j K\right)\right)^{2} \mathbb{E}\left[\left(W_{(i)}(K)\right)^{2}\right]+O\left(M^{2} L^{3 \xi-2}\right) \\
= & \left\|Z\left(s_{0}+j K\right)\right\|^{2} K+O\left(M^{2} L^{3 \xi-2}\right) .
\end{aligned}
$$

We conclude that

$$
\mathbb{E}\left(\left(\mathfrak{x}_{j+1}-\mathfrak{x}_{j}\right) 1_{\bar{s}>j K}\right)=O\left(L^{3 \xi} M^{-3}+L^{\xi-2}\right) .
$$

Thus

$$
\mathbb{E}\left(\mathfrak{x}_{\min \{\bar{s} / K, A / K\}}\right)=2 m+O\left(A L^{2 \xi} M^{-3}+A_{M} L^{-2}\right)
$$

Recall that we are given some $\gamma<1$. 
- In case of Lemma 6(b), let us choose $A=M^{2+\frac{1-\gamma}{\gamma}}$. Assuming, as we can that $\xi<1-\gamma$ gives $A L^{-2}<L^{-\xi}$. If $\xi$ is small (say smaller than $1 / 20$ ), then $A L^{2 \xi} M^{-3}<L^{-\xi}$ holds as well.

- In case of Lemma 6(c) let us choose $A=L^{2} \log ^{-3 / 2} L$. Since both $1-\gamma$ and $\xi$ are small, we have $A L^{2 \xi} M^{-3}+A_{M} L^{-2}=O\left(\log ^{-3 / 2} L\right)$.

Thus the error term in (24) can be replaced by $O(E)$, where $E=L^{-\xi}$ in case of Lemma 6(b) and $E=\log ^{-3 / 2} L$ in case of Lemma 6)(c). Next, by Lemma 5,

$$
\mathfrak{x}_{\min \{\bar{s} / K, A / K\}}=2 m \pm 2+O\left(L^{\xi} M^{-1}\right)=2 m \pm 2+O\left(L^{-\xi}\right)
$$

holds except on a set of probability $O\left(\theta^{A / M^{2}}\right)$. On this exceptional set, we bound the left hand side of (25) by $O(\log M)$. In case of Lemma 6(b), $\theta^{A / M^{2}} \log M$ is superpolynomially small in $L$. In case of Lemma 6)(c), $\theta^{A / M^{2}} \log M<$ $\theta^{\sqrt{\log L}} \log L=O\left(\log ^{-3 / 2} L\right)$. It follows that

$$
\mathbb{P}\left(\left\|Z_{s_{0}+\bar{s} K}\right\| \leq M / 2-K\right)=\frac{1}{2}-O(E)
$$

Since $\left\|Z_{s_{0}+\bar{s} K}\right\| \leq M / 2-K$ implies $Z\left(s_{1}\right) \in \mathcal{C}_{m-1}$, we conclude

$$
\mathbb{P}\left(Z\left(s_{1}\right) \in \mathcal{C}_{m-1}\right) \geq \frac{1}{2}-O(E) .
$$

An analogous argument shows

$$
\mathbb{P}\left(Z\left(s_{1}\right) \in \mathcal{C}_{m+1}\right) \geq \frac{1}{2}-O(E)
$$

Lemma 6(b) and (c) follow.

\section{ACKNOWLEDGEMENTS}

I am highly indebted to Imre Péter Tóth for raising a question which initiated this research. I am also grateful to Lai-Sang Young for discussions and encouragement. This project began while I was affiliated with the Courant Institute, New York University.

\section{REFERENCES}

[A78] Anulova, S. V., Diffusion process with singular characteristics, International symposium on stochastic differential equations, Vilnius, 7-11 (1978).

[BGNSzT15] Bálint, P., Gilbert, T., Nándori, P., Szász, D., Tóth, I. P., On the limiting Markov process of energy exchanges in a rarely interacting ball-piston gas Preprint http://arxiv.org/abs/1510.06408 (2015).

[B68] Billingsley, P., Convergence of probability measures, Wiley (1968).

[CHS81] Cambanis, S., Huang, S., Simons, G., On the theory of elliptically contoured distributions, Journal of Multivariate Analysis 113 368-385 (1981).

[CGRS15] Carinci, G., Giardinà, C., Redig, F., Sasamoto, T., Asymmetric stochastic transport models with $\mathcal{U}_{q}(\mathfrak{s u}(1,1))$ symmetry Preprint http://arxiv.org/pdf/1507.01478.pdf (2015). 
[ChShY04] Cherny, A., Shiryaev, A. and Yor, M. (2004). Limit behav ior of the "horizontalvertial" random walk and some extension of the Donsker-Prokhorov invariance principle. Theory Probab. Appl. 473 377-394 (2004).

[DSzV08] Dolgopyat, D., Szász, D., Varjú, T., Recurrence properties of Lorentz gas, Duke Math. J. 142 241-281 (2008).

[KMP82] C. Kipnis, C. Marchioro, E. Presutti: Heat flow in an exactly solvable model Journal of Stat. Phys. 27 65-74 (1982)

[K14] Klenke, A., Probability Theory, Universitext, 2014.

[L06] Lejay, A., On the constructions of the skew Brownian motion, Probability Surveys 3 413-466 (2006).

[LNY15] Li, Y., Nándori, P., Young L-S., Local thermal equilibrium for certain stochastic models of heat transport preprint (2015).

[P90] Portenko, N. I. Generalized diffusion processes. Translations of Mathematical Monographs 83 American Mathematical Society (1990).

[RY07] Ravishankar, K., Young, L.-S., Local Thermodynamic Equilibrium for some Models of Hamiltonian Origin Journal of Stat. Phys. 1283 (2007)

[RY99] Revuz, D., Yor, M., Continuous Martingales and Brownian Motion, Grundlehren der mathematischen Wissenschaften 1999.

[SSz04] Sidoravicius, V., Sznitman, A.-L., Quenched invariance principles for walks on clusters of percolation or among random conductances, Probability theory and related fields 1292 219-244 (2004).

[SV07] Stroock, D., W, Varadhan, S.R.S., Multidimensional Diffusion Processes, Springer, 2007.

[Z83] Zessin, H., The Method of Moments for Random Measures, Z. Wahrscheinlichkeitstheorie verw. Gebiete 62 395-409 (1983).

Péter Nándori: Department of Mathematics, University of Maryland, ColLEGE PARK, MD 20742, USA

E-mail address: pnandori@math.umd.edu 